\title{
ALL'INDOMANI DEI PATTI LATERANENSI (1929) LE REAZIONI DEI CATTOLICI ITALIANI
}

\author{
ANNIBALE ZAMBARBIERI (*) \\ Nota presentata dal m.e. Silvio Beretta \\ (Adunanza del 19 dicembre 2019)
}

\begin{abstract}
SunTO. - Premessa una breve panoramica sulle aspettative del mondo cattolico italiano verso la soluzione della Questione Romana, lo studio effettua una rassegna delle reazioni alla firma dei Patti Lateranensi da parte del clero e del laicato. Risaltano le approvazioni entusiaste di molti, che videro nell'evento sia la fine di annosi contrasti tra Chiesa e Stato, sia il raggiungimento di un proficuo accordo con il regime fascista. $\mathrm{Pa}$ rallelamente non mancarono, pur minoritarie, le riserve e le delusioni, soprattutto di esponenti del disciolto Partito Popolare Italiano, che ravvisavano in quelle intese un indebito appoggio all'establishment politico. Altri prospettarono mutamenti nei programmi dell'associazionismo, indirizzandoli a formare culturalmente e spiritualmente soprattutto le élites dei fedeli. Un certo rilievo ebbero, dopo i discorsi di Mussolini alla Camera dei deputati e al Senato, nel corso delle procedure per la ratifica dei Patti, le prese di distanza dalle parole del capo del governo, che tendevano a rivendicare la superiorità dello Stato sulla Chiesa. Le relative tensioni vennero allentate, inaugurando una concorde collaborazione, non scevra tuttavia di momentanei attriti. Comunque il tentativo del regime di configurare una propria religione dovette stemperarsi di fronte al persistere di convincimenti e di pratiche cultuali radicati sul lungo periodo.
\end{abstract}

$* * *$

ABSTRACT. - After an overview of the Italian Catholics expectations towards the solution of the Questione Romana, this study carries out a review of the reactions to the signing of the Patti Lateranensi from the clergy and laity. We will see the endorsements of many, who saw in the event the end of years of contrasts between Church and State, both the achievement of an agreement with the fascist regime. At the same time, not the reservations and disappointments were lacking, even though they were minority of exponents of the dissolved Partito Popolare, which saw in those arrangements an undue support to the political establishment; others predicted changes in the programs of the

(*) Università degli Studi di Pavia, Italy. E-mail: zaman@unipv.it 
Catholic organizations, directing them to form culturally and spiritually especially the élite members of the laity. After Mussolini's speeches in the Camera dei deputati and in the Senato, on the procedures for the ratification of the Patti, the claim of the State's superiority over the Church were viewed with concern. The tensions were loosened, inaugurating a consensus cooperation, but not free of momentary friction. However, the regime's attempt to configure its own religion had to fade away in the face of the persistence of convictions and long-established cultic practices.

1. Per introdurre in modo disinvolto qualche nota sui commenti dei cattolici italiani alla stipulazione dei Patti Lateranensi, sia consentito ricorrere ad una metafora tratta dalla meteorologia. La scelta non è del tutto arbitraria, perché se ne avvalse anni dopo, commemorando il fatto, un testimone memore anche dei rigori di quell'inverno. In particolare durante il febbraio del 1929, quando vennero sanciti gli accordi, un'ondata di freddo polare si abbatté sull'Italia come sul resto dell'Europa. Senza ricorrere ai dati dell'Ufficio Presagi, come allora si designava il servizio nazionale per le previsioni del tempo, un rapido riscontro del fenomeno risalta dalle cronache dei quotidiani di quelle settimane che indicavano frequentemente temperature inferiori allo zero insieme a forti precipitazioni nevose, con il corredo di parecchie fotografie. Il "Corriere della Sera" titolava l'11 febbraio Ancora giornate glaciali in Europa, segnalando un'abbondante nevicata a Roma e naturalmente a Milano, anche se questa città era stata l'ultima «contro ogni tradizione a ricevere l'omaggio della bianca visitatrice». Né mancava una curiosa sorpresa: la superficie del lago di Pusiano (il «vago Eupili mio» di Parini), era ricoperta da una lastra di ghiaccio così spessa e resistente da riuscire a sostenere il peso e la corsa di una motocicletta tra le località di Castelletto e appunto Pusiano. In effetti ricordando trent'anni più tardi l'anniversario dei Patti, un religioso destinato a buona notorietà, evocava l'evento, correlandolo al disagio, fisico per il clima inclemente, e psicologico a motivo della sua sofferta valutazione. L'esperienza restava per lui ancora vivida, alla luce dei successivi avvenimenti. L'esordio riecheggia il motivo del freddo pungente: «Nel lontano febbraio del ventinove, il gelo eccezionale segnato dai barometri si dimostrò ancora inadeguato ad esprimere il gelo che invase gli spiriti all'annuncio, inatteso e clamoroso, dei conclusi patti lateranensi. Né euforie di sfere ufficiali, né apologie di stampa imbavagliata, né un clima di viltà e di dimissione, valsero a ridurre o a scalfire un'opposizione che coalizzava imponenti forze di provenienza e finalità contrastanti. I laicisti ravvisarono, nella pacificazione tra Chiesa e Stato, la disfatta più irreparabile di tutte le loro conquiste in quattro secoli di lotte; la grande maggioranza dei 
cattolici non riusciva a rendersi ragione come la Chiesa avesse potuto venire a patti con una forza dimostratasi anticristiana in sé, nei fini come nei mezzi; le masse fasciste [...] intuirono opposizioni di fondo, inconciliabilità di programmi». ${ }^{1}$ La prosa veicola le impressioni che lo scrivente, $\mathrm{p}$. Giulio Bevilacqua , sacerdote oratoriano dell'istituto La Pace di Brescia, confessa di aver provato in quel febbraio 1929. Come ogni ricordo, anche questo risente di situazioni e di riflessioni posteriori, ma con ogni probabilità ribadisce un'esperienza che rimase incisa nella sensibilità del religioso. Lo storico Mario Bendiscioli , frequentatore del circolo di cattolici aperto alla Pace, scriverà più tardi che un religioso, di cui non faceva il nome, ma chiaramente identificabile in Bevilacqua, aveva indirizzato nel 1926 al direttore del quotidiano fascista locale una dura protesta pubblicata contemporaneamente sul foglio cattolico "Il Cittadino di Brescia", per condannare i comportamenti dei seguaci di Mussolini. La rivalsa non si era fatta attendere e il giornale cattolico «pagava l'ardimento della pubblicazione col rogo e l'autore quello d'averla scritta con l'esilio dalla città». ${ }^{2}$ Bevilacqua fu costretto a trasferirsi a Roma dove tuttavia ebbe agio di intensificare la sua familiarità con l'amico di cui era consigliere sprituale, il bresciano Giovanni Battista Montini, allora minutante nella Segreteria di Stato Vaticana. Come si sa quest'ultimo, diventato papa, lo nominerà vescovo e cardinale nel 1965 permettendogli di continuare a svolgere il ministero in una parrocchia alla periferia di Brescia. ${ }^{3}$

1 G. BEVILACQUA, I Patti Lateranensi, dopo trent'anni, in "Humanitas", 14 (1949), pp. 182-190, qui p.182.

2 M. BENDISCIOLI, Antifascismo e Resistenza, Roma 1964, p.140. Sul personaggio si veda A. FAPPANI, Bevilacqua Giulio (1881-1965), in F. TRANIELLO, G. CAMPANINI (edd.), Dizionario storico del movimento cattolico in Italia. 1860-1980 [d'ora in poi: DSMCI], II. I protagonisti, Casale Monferrato 1982, pp. 43-45. Lo stesso Fappani ne ha scritto una biografia, Padre Giulio Bevilacqua. Il Cardinale parroco, Brescia, 1979. Si legga anche F. MOLINARI, Padre Giulio Bevilacqua e il fascismo, in Momenti e aspetti della cultura cattolica nel ventennio fascista, Brescia 1977, pp.29-57. Per una ricostruzione più ampia dei suoi orientamenti, G. STELLA, Il pensiero religioso di Giulio Bevilacqua. Alcuni motivi, in G. GHIDELLI (ed.), Teologia, liturgia, storia. Miscellanea in onore di Carlo Manziana, Brescia 1978, pp. 411-450.

3 Sul rapporto tra i due, reso più stretto a Roma dal 1928, si veda R.MORO, Giovanni Battista Montini e il fascismo, in Paul VI et la modernitè dans l'Eglise, Rome 1984, pp.51-52. Particolarmente significativo il breve profilo che gli dedicò lo stesso MONTINI, Bevilacqua. Ottant'anni, in "“Humanitas”, 26 (1961) pp. 777-781 (molto interessante l'intero numero, giugno-luglio 1965 della stessa rivista, Scritti e testimonianze di p. Giulio Bevilacqua, cardinale). 
Sull'abbrivio ispirato alla metafora termica esordisce anche un brano dell'opera di un altro testimone, Arturo Carlo Jemolo, aduso nei suoi contributi scientifici ad inquadrare episodi, tendenze, personaggi, in un'ottica storiografica, con equilibrio e notevole acutezza. Nel volume, ormai un classico, Chiesa e Stato in Italia negli ultimi cento anni, da lui pubblicato nel 1948 e più volte riedito e aggiornato, riferisce impressioni diverse, se non opposte, rispetto a quelle descritte dal sacerdote bresciano. I cenni riattualizzano, con l'uso del presente, una differente temperie: «Più calorosa contentezza tra i cattolici: a prescindere dall' ostentato entusiasmo dei cattolici non compromessi irrimediabilmente con il fascismo, ma rimasti fino allora in un'attitudine di freddezza, che colgono l'occasione per inserirsi, sta la reale gioia di coloro per i quali pure la traccia di quel dissenso pesava, non solo per il piccolo numero d'imbarazzi e di situazioni equivoche che ancora poteva loro procurare, ma perché in effetto ai loro occhi una bandiera non benedetta dal papa era una bandiera che cedeva in dignità alle altre. In molti di questi v'è, forse inconscia, la vecchia tenace idea storica di una posizione peculiare dell'Italia, di un suo legame intimo con la Santa Sede [...]. Gli Accordi lateranensi debbono ai loro occhi valere a riannodare sotto questo riguardo una tradizione millenaria». ${ }^{4}$ Nei due ricordi, dunque, la difforme valutazione, o per meglio dire una caratterizzazione dissimmetrica, fa arguire il moltiplicarsi di itinerari interpretativi, e per la verità anche operativi a riguardo dei fatti ricordati.

2. Alla rassegna di reazioni e giudizi, è opportuno premettere una breve panoramica sulle aspettative che nel mondo cattolico precedettero l'evento del 1929. L'obbligato background è costituito dalla cosiddetta "questione romana". Il binomio designa un ispido tessuto di polemiche, contrapposizioni, divisioni all'esterno e all'interno della compagine dei cattolici, ma anche tra intellettuali di diverso orientamento. Molti fattori alimentarono le controversie. Si possono enumerare in un elenco allusivo: la famosa legge delle Guarentigie promulgata dal governo italiano nel 1870 dopo la presa di Roma da parte dell'esercito piemontese; i deliberati del Concilio Vaticano I nello stesso anno; il venta-

4 A.C. JEMOLO, Chiesa e Stato in Italia negli ultimi cento anni, Torino 1963 ( nuova edizione riveduta e ampliata), p.468. 
glio dispiegato dalle opzioni politiche e dalle tendenze ideali; i tentativi di riforma ecclesiastica; i moti anticlericali che punteggiarono la storia del Paese dopo la raggiunta unità e oltre l'alba del XX secolo. La prima guerra mondiale, spartiacque tragico e decisivo, ebbe ripercussioni anche sull'ormai annoso problema. Infatti, terminate le ostilità, s'intravidero spiragli per sciogliere alcuni nodi del contenzioso. Un iter al riguardo sembrò aprirsi mediante alcuni colloqui intrattenuti a Versailles in margine alla Conferenza di pace, tra il sacerdote statunitense Francis C. Kelley e il marchese Brambilla, consigliere della commissione italiana ai negoziati, e in seguito tra quest'ultimo e Bonaventura Cerretti, della vaticana Congregazione degli Affari Straordinari, presente nella capitale francese. Durante un privato rendez-vous con il presidente del Consiglio Vittorio Emanuele Orlando, il prelato comunico una nota del Segretario di Stato Vaticano card. Pietro Gasparri. Vi si avanzava l'ipotesi di costituire un piccolo Stato Vaticano, quale nuova entità politico-giuridica, autonoma dallo Stato Italiano, dove il Pontefice potesse esercitare la sua missione spirituale e temporale per la Chiesa. Nel colloquio, a quanto pare, veniva introdotta con discrezione l'idea di rimodellare la legislazione italiana in materia ecclesiastica. Come ormai è stato acclarato, gli scambi di vedute non sortirono effetto per l'atteggiamento di Vittorio Emanuele III. Stando all'informazione del vicepresidente del Consiglio, on. Gaspare Colosimo, diretta al presidente Orlando in una lettera del giugno 1919, il sovrano riteneva che la conciliazione con la Santa Sede avrebbe annullato «tutti i benefici di tante lotte culminate con la legge delle

5 Oltre all'opera di Jemolo appena citata, che offre un'ampia ricostruzione dei fatti (pp.173-412), è utile rivedere l'affresco, con scorci e profondità di vedute offerto da F.CHABOD, Storia della politica estera italiana dal 1870 al 1896, Bari 1962. Molti dati in V. DEL GIUDICE, La Questione romana e i rapporti tra Stato e Chiesa prima della Conciliazione, Roma 1947.

6 F. MARGIOTTA BROGLIO, Italia e S. Sede dalla Grande Guerra alla Conciliazione, Bari 1966, p. 366. Omettendo numerosi riferimenti bibliografici, ci si limita a rinviare alla puntuale sintesi di P.DORIA, Il molo di Gaspare Colosimo e del re nel rifiuto della bozza Gasparri, in A. MELLONI, G. CAVAGNINI, G. GRASSI, Benedetto XV, Papa Giacomo Della Chiesa nel mondo dell'inutile strage, Bologna 2017, pp. 654-666. Sulla partecipazione non ufficiale di Cerretti al negoziato parigino, mi permetto di rinviare al mio saggio dal titolo La pace di Versailles, il Giappone, il Vaticano, di prossima pubblicazione presso l'Istituto Veneto di Scienze Lettere ed Arti, negli Atti del convegno tenuto a Venezia il 15-16 novembre 2018 dal titolo 1919-1920. I trattati di pace in Europa. 
Guarentigie»; anzi, soggiungeva che il re «andrebbe via, piuttosto che sobbarcarsi ad un concordato somigliante». ${ }^{6} \mathrm{Va}$ ribadito come non fosse assodato che le avances mirassero oltre ad un Trattato, anche esplicitamente ad un Concordato. Di fatto dopo quella chiusura si reiterarono sollecitazioni volte ad uscire dall'impasse, in vista di assicurare una autonomia alla Sede Pontificia, che concretizzasse in modo stabile l'arguto pronostico espresso anni prima da Ignaz von Döllinger. Questo sacerdote e storico duramente critico nei confronti dei pronunciamenti del Vaticano riguardo al ruolo e alle prerogative del vescovo di Roma, nel 1861 aveva scritto che in futuro si sarebbe sicuramente ovviato alla situazione critica in cui era venuto a trovarsi il pontefice. Per rendere la previsione, ricorse alla mitologica nascita di Apollo, quando, per dar un asilo al nuovo dio, emerse dal mare l'isola di Delo. Senza dubbio, a suo dire, non sarebbe mancata, al soglio di Pietro, «la sua Delo», anche se avesse dovuto emergere con un balzo dal mare. ${ }^{7}$ Come ben si sapeva e come meglio si seppe in seguito, il miracoloso auspicio stentò a realizzarsi e le idee al riguardo si susseguirono numerose e confuse. Basti menzionare due, esposte da un ecclesiastico dopo la conclusione del conflitto mondiale, poco conosciute nelle ricostruzioni storiografiche sul tema, ma che a suo tempo riscossero una discreta udienza. Nel biennio 1920-1921 polemiche sollevò l'intervento di Adriano Bernareggi, colto sacerdote milanese distintosi per le sue posizioni in campo liturgico e pastorale, destinato in seguito a diventare vescovo di Bergamo. ${ }^{8}$ La sua opinione prendeva un certo risalto dai ruoli di responsabilità che egli ricopriva in qualità di docente, sia nel seminario arcivescovile milanese sia nella neonata Università Cattolica del Sacro Cuore alla cui fondazione aveva contribuito come membro del comitato promotore. In

7 J.I. von DÖLLINGER, Kirche und Kirchen, Papstthum und Kirchenstaat, München 1861, p.684: «Als ein neuer Gott, habe geboren warden sollem, da sei die Insel Delos aus dem Meer emporgestiegen, um dem Gott als Geburtstätte zu dienen. Wir konnen zuversichtlich erwarten dass, was auch kommen möge, dem Stuhle Petri ein Delos nich fehlen werde, und sollte es ersts aus den Meere emporsteigen». Su questo personaggio (1799-1890) si vedaTH. A.HOWARD, The Pope and the professor: Pius IX, Ignaz von Döllinger, and the quandary of modern age, Oxford 2017.

8 R. AMADEI, Bernareggi Adriano (1884-1953), in DSMCI, vol.II. I protagonisti, pp.37-40. Si veda anche la recente pubblicazione di A. A. PERSICO, "Consul Dei”. Adriano Bernareggi (1884-1953). Chiesa e società nell'Italia del Novecento, Roma 2019, 3 voll: per la questione qui trattata, vol. I, pp.186-203. 
due articoli apparsi sulla rivista "Vita e Pensiero", redatta da protagonisti dell'esordio del nuovo ateneo, egli aveva innanzitutto rivisitato, in un'ottica non polemica, gli eventi relativi all'unificazione politica italiana. ${ }^{9}$ L'autore, poiché a suo dire la funzione storica del potere temporale poteva ritenersi esaurita, opinava che il tema dell'autonomia territoriale della sede del papato potesse più pacificamente essere affrontato esulando dall'ambito del dogma: « dogmatica è soltanto - asseriva- la questione del diritto del pontefice di garantire la propria indipendenza spirituale: questo diritto è immutabile e uniforme sempre, ma non è punto immutabile né sempre uniforme il modo di concretarlo». Era questa una recisa presa di distanza dai corollari che, nelle dispute teologiche, si desumevano dalla connessione tra la sede romana del pontefice e il primato petrino. ${ }^{10} \mathrm{Al}$ di là delle osservazioni sugli equivoci del passato e qualche riserva sulla condotta di Pio IX, Bernareggi esponeva istanze per una riconfigurazione del posizionamento della S. Sede in Italia. Sia all'interno della diocesi milanese come da parte di esponenti dell'episcopato e soprattutto del gesuita Enrico Rosa, scrittore de "La Civiltà Cattolica", così come del card. Prefetto della Congregazione dei Seminari e delle Università, molte critiche vennero rivolte a questo articolo. Grazie anche ai buoni uffici del neo arcivescovo ambrosiano Achille Ratti, l'autore poté spiegarsi in un nuovo studio pubblicato nel numero di settembre 1921 della stessa rivista. Sfumate le osservazioni riguardanti Pio IX, passava ad elencare alcune opinioni di autori cattolici, asserendo da parte sua che lo Stato italiano avrebbe dovuto riconoscere «al romano pontefice una sovranità effettiva e propria». Ciò esigeva, beninteso, che il papa potesse «trattare e non da inferiore, con qualunque governo, l'italiano compreso». Non sarebbero mancate difficoltà per ottenere tale risultato. E tuttavia Bernareggi concludeva, speranzoso: «ma perché anche queste ultime difficoltà non potranno essere vinte in un giorno non lontano?». ${ }^{11}$

Che nuovi approcci alla problematica si potessero allora auspica-

9 A. BERNAREGGI, Il Papato e il problema nazionale italiano (nel $50^{\circ}$ della breccia di Porta Pia), in "Vita e Pensiero", 6 (1920), pp.522-535, 629-637.

10 Si veda in proposito il mio saggio Roma "Romanitas": un'ecclesiologia della visibilità e dell'autorità nel secondo Ottocento, in A. ZAMBARBIERI (ed), I cattolici e lo Stato liberale nell'età di Leone XIII, Venezia 2008, pp. 69-118, spec. 78-103.

11 A. BERNAREGGI, Cinquant'anni di prova della legge delle Guarentigie (1871-1921), in "Vita e Pensiero", 7 (1921), pp. 524-548, cit. alle pp. 544-548. 
re, o prevedere, trapelava da avvisaglie, variamente percepite anche in rispondenza alla situazione generale del Paese. Se il Partito Popolare di don Sturzo da poco fondato conferiva un peso ragguardevole alla presenza politica dei cattolici, il movimento fascista andava progressivamente abbandonando l'iniziale anticlericalismo e pure i progetti volti sia a sequestrare i beni delle congregazioni religiose sia ad abolire le mense vescovili. Nuove disponibilità per un proficuo dialogo sembrarono aprirsi ai vertici dei poteri religiosi e civili. Quasi in concomitanza con l'uscita dell'ultimo articolo di Bernareggi, due quotidiani, "Il Messaggero" e "Il Secolo" in data 19 settembre 1921 pubblicavano l'intervista che il sacerdote Ernesto Buonaiuti, professore di Storia del Cristianesimo all'Università di Roma, aveva ottenuto da un «cardinale» di cui non si faceva il nome ma che era facilmente identificabile in Gasparri, Segretario di Stato Vaticano. Il testo, nello stile tipico dell'autore, capace di adeguarsi al taglio giornalistico, grazie alla struttura ben compaginata di botte e risposte, insieme a qualche slargo descrittivo, riferiva valutazioni abbastanza esplicite del porporato. Il quale constatava con favore che s'era fatta strada, anche in partiti distanti dal sentire cattolico, l'idea di una fuoruscita dalla questione romana. Ma «imprescindibile e incontrovertibile» per lui era la necessità «che alla S. Sede, per l'esercizio sovrano del suo potere mondiale», fosse garantita «una completa indipendenza, non solamente reale, ma anche apparente $[. .$. legata ad un possesso territoriale, di qualsiasi proporzione».

Buonaiuti dovette poi ammettere che Gasparri reagì negativamente alla pubblicazione giornalistica, con il risultato di incrinare il rapporto tra i due. Probabilmente gli rimproverava di aver propalato alcune indiscrezioni, come le riserve nei confronti del Partito Popolare o giudizi su uomini politici. Inoltre la frase finale risentiva di un'inflessione tipicamente buonaiutiana, forse non gradita al suo interlocutore, perché vi si invitavano tutte le «anime religiose del mondo» a comprendere come Cristo dovesse essere servito «sul terreno della religiosità e dell'unione, non su quello della politica e delle rivalità confessionali». La frase era suscettibile di un'interpretazione in senso sia ecumenico sia eccessivamente refrattario a trattative con il mondo politico. ${ }^{12}$

Il colore mediatico, più o meno aderente al pensiero del porpora-

12 L'intervista è riportata in BUONAIUTI, Pellegrino di Roma, Bari $1964^{2}$, pp.190-194 (i corsivi delle due parole reale e apparente sono nel testo dell'intervista). 
to, lasciava comunque intendere come la situazione fosse fluida né si escludesse la possibilità di un'interlocuzione tra Gasparri e il capo del fascismo. E forse ancor prima dell'intervista rilasciata a Buonaiuti, il porporato, avrebbe incontrato il duce, quantunque il fatto sia difficile da documentare, nell'estate del 1921, anteriormente alla marcia su Roma. Comunque, dopo l'ingresso dei fascisti nella capitale, si può collocare con sufficiente approssimazione nel novembre 1922 l'iniziativa di Mussolini per un abboccamento con il porporato. Di sicuro l'appuntamento si ebbe nel gennaio dell'anno successivo, grazie all'ospitalità offerta dal conte Carlo Santucci, a palazzo Guglielmi, provvisto di due ingressi, in via del Gesù e in via della Pigna, e perciò gli interlocutori poterono entrare separatamente, in modo da evitare la benché minima notorietà all'incontro. ${ }^{13}$ Pare che i due personaggi non abbiano parlato in termini precisi né di Trattato né di Concordato, ma intanto si erano stabiliti i prodromi di futuri sviluppi e abbozzi di concreti progetti, come risulta da un ricordo dell'ambasciatore francese François CharlesRoux. ${ }^{14}$

Occorre non dimenticare altri approcci di cattolici verso il capo del fascismo, e più in generale le precoci simpatie che essi nutrivano nei confronti del movimento. Tale propensione emerge, quasi in un fermo immagine, dallo spettacolo cui assistette con sgomento Luigi Sturzo che il 30 ottobre 1922 assistette allo snodarsi della marcia su Roma nelle vie della capitale mentre si trovava nella villa Ruffo Della Scaletta. Il sacerdote, comprensibilmente sconvolto, vide sfilare insieme alle squadre fasciste parecchi preti dietro i labari recanti immagini di teschi. ${ }^{15}$ Non aveva bisogno di quella vista per confermare la sua avversione al movimento mussoliniano. Ma altri esponenti del suo partito entrarono a far parte del primo ministero presieduto dal capo del fascismo. Tra questi

13 MARGIOTTA BROGLIO, Italia e S. Sede, pp.107-110; si veda anche l'appunto dello stesso Santucci pubblicato alle pp.441-443.

14 Secondo confidenze a lui fatte da Gasparri, « le chef fasciste s'étant d'emblée montré disposé à reconnaitre au Pape une souveraineté temporelle sur une parceille de Rome. - Mais, lui avait fait observer le Cardinal, vous ne pourriez pas faire voter cela à la chambre - eh bien, avait répondu Mussolini, l'on changera la chambre. - Mais si vous changez la chambre sans changer la loi électorale, les électeurs vous renderont une chambre semblable à l'ancienne - Eh, bien avait répliqué Mussolini l'on changera la loi électorale»: F. CHARLES-ROUX, Huit ans au Vatican. 1932-1940, Paris 1947, pp. 47-48.

15 G DE ROSA, Il Partito Popolare italiano, Bari 1972 pp. 183-184, in nota. 
l'avvocato Fulvio Milani che, una volta simpatizzante di Romolo Murri, aveva un consistente seguito nel Partito Popolare, come dimostrarono i voti ottenuti nel giugno 1919 al convegno di Bologna, di numero inferiore solo a quelli ottenuti del sacerdote di Caltagirone..$^{16}$ Ricoprì un ruolo non secondario per le questioni ecclesiastiche, essendo sottosegretario al ministero di Grazia e Giustizia e Affari di Culto. ${ }^{17}$ Al lui si rivolse in quel torno di tempo il sacerdote Giovanni Minozzi, distintosi nella prima guerra mondiale come fondatore e direttore delle case del soldato, punti di ristoro e di ritrovo dove i combattenti in prima linea potessero riprendere le forze ${ }^{18}$ Egli aveva stretto molti legami durante il conflitto con politici della corrente nazionalista. In un suo memoriale, ancora inedito, ragguaglia sulle iniziative che prese verso la fine del 1922. Vi narra come, tramite Milani, avesse preso contatto sia con il titolare del suo ministero Aldo Oviglio, da lui ritenuto «un cattolico praticante, ma italiano autentico, aperto di mente», sia con Amedeo Giannini direttore generale al Ministero degli Esteri, «cattolico integrale, sul serio fedelissimo alla Chiesa e alla Patria». Insieme si incontrarono con padre Giovanni Genocchi, dei Missionari del Sacro Cuore la cui sede era sita in via del Risorgimento, davanti a S. Ivo alla Sapienza, quindi prossima ai palazzi delle istituzioni parlamentari. Il religioso godeva di considerevole prestigio, sia per la sua competenza in materia di studi storico-critici, sia per la conoscenza del mondo extraeuropeo, essendo stato missionario in Nuova Guinea. Nonostante le sue amicizie con $i$ "modernisti", era ben introdotto negli ambienti della Curia vaticana, tanto che venne incaricato di un'inchiesta sulla schiavitù nell'America del Sud, utilizzata da Pio X. ${ }^{19}$ Nei loro rendez-vous queste persone irrobustirono il loro convincimento che ormai i tempi fossero maturi, secondo le parole di Milani, «per trattare con la S. Sede per conclusioni radicali, definitive». Anche il barnabita Giovanni Semeria, molto legato sia a Minozzi che a Genocchi, aveva preso parte a queste

16 Ibid. p.29.

17 F. MALGERI, Milani Fulvio 1885-1945, in DSMCI III/2 (1984), pp. 560-561.

18 Si rimanda al mio saggio Le "Case del soldato alla fronte»: note sull' iniziativa di don Giovanni Minozzi, in F.BIANCHI - G. VECCHIO (edd), Chiese e popoli delle Venezie nella Grande Guerra, Roma 2016, pp. 235-266.

19 L. BEDESCHI, Genocchi Giovanni, in DSMCI III/1 (1984), pp. 403-404 e dello stesso I pionieri della DC 1896-1906, Milano 1966, pp. 277-279; F. TURVASI, G. Genocchi e la controversia modernista, Roma 1974. 
riunioni. Così, nel gennaio del 1923, padre Genocchi poté presentare una relazione sugli avvenuti colloqui al segretario di Stato Gasparri. Il quale si mostrò «guardingo, da politico alla Giolitti», avanzando qualche riserva prudenziale («non si prendono le noci nel sacco») ed infine lasciò trasparire il proprio appoggio a ventilati sviluppi negoziali, ché il suo volto «s'accese d'un sorriso compiaciuto e furbo». ${ }^{20}$

Parve un inizio lusinghiero, quantunque sia Genocchi che Minozzi e Semeria, pur continuando a coltivare buoni rapporti con l'establishment politico, passassero in secondo piano, lasciando il ruolo del diretto interlocutore con Mussolini al gesuita Pietro Tacchi Venturi, ragguardevole studioso che vantava aderenze cospicue, avendo svolto l'incarico di Segretario generale della Compagnia dal 1914 al 1921, tanto più che nel corso della guerra supplì il Generale Wladimiro Ledochowshi, suddito austriaco e perciò allontanato da Roma. ${ }^{21}$ Poche indiscrezioni varcarono le stanze della diplomazia e comunque l'opinione pubblica non era in grado di seguire il dipanarsi di quegli approcci. Tuttavia, nonostante l'alternarsi delle violenze fasciste, Mussolini pareva erigersi via via «a unico alfiere degli interessi della Chiesa». ${ }^{22}$ Da non trascurare il fatto che al capo del Fascismo il presidente dell'Azione Cattolica Italiana Luigi Colombo in data 18 gennaio 1923, quindi uno o due giorni prima dell'incontro Mussolini-Gasparri a palazzo Guglielmi, aveva chiesto al governo di recepire le istanze cattoliche su problemi come l'insegnamento religioso nelle scuole, la pubblica moralità, il riconoscimento civico delle feste di precetto. ${ }^{23}$ A diffondere la sensazione di una compatibilità tra fascismo e Chiesa cattolica, contri-

20 G. MINOZZI, Fra i prodromi della Conciliazione, ms. di 3 cc,in Archivio della Congregazione Famiglia dei Discepoli, F. Minozzi/ opere / 112.

21 G. SALE, Fascismo e Vaticano prima della Conciliazione, Milano 2007, pp. 35-37; R. PERTICI, Chiesa e Stato in Italia. Dalla Grande Guerra al nuovo Concordato (1914-1984), Bologna 2009, p.104, n.12. Per un breve profilo del gesuita si veda S. TRAMONTIN Tacchi Venturi, Pietro (1881-1956), in DSMCI II, pp. 631-633.

22 L. CECI, L'interesse superiore. Il Vaticano e l'Italia di Mussolini, Bologna 2013, p.85 e per vari interventi in favore della Chiesa, pp.91-123. Sintomatico quanto già nel 1924 Ludwig von Pastor, rappresentante in Vaticano della Repubblica austria$\mathrm{ca}$, scrisse in un suo rapporto diplomatico per sottolineare come molti riconoscessero a Mussolini il merito di aver ridato «all'autorità e alla religione nella vita politica italiana il posto che loro spettavano» (F.ENGEL-JANOSI, Il Vaticano fra Fascismo e Nazismo, Firenze 1973, p. 71)

23 A. ZAMBARBIERI, Colombo Luigi, in DSMCI, II, p.115. 
buirono l'esaudimento dei desiderata esposti da Colombo, ma anche altri provvedimenti, quali l'aumento dei contributi economici per il clero e il ripristino di segni cristiani come il crocefisso nei locali pubblici. Non pochi fedeli guardarono con evidente simpatia a queste misure e ai programmi di opposizione al socialismo, che i fascisti adottarono grazie anche all'alleanza, quantunque meno chiaramente percepita, con il capitale agrario e industriale. Si veniva già instaurando in forma sempre più netta un regime dai tratti progressivamente più distinguibili, specie dopo il discorso tenuto alla Camera il 3 gennaio 1925, nel quale Mussolini si attribuì la responsabilità su tutte le azioni che lo avevano portato alla conquista del potere. ${ }^{24}$ Linfa al conformismo crescente veicolavano alcune riproposizioni, dal versante politico, di motivi devozionali, quale il culto a S. Francesco. Per la ricorrenza anniversaria dei settecento anni dalla morte del Santo, la congiunta partecipazione del ministro Pietro Fedele, rappresentante del governo, e del card. Merry del Val, legato pontificio alle celebrazioni tenute ad Assisi, pare rendesse celeri i passi verso trattative in vista di accordi tra Italia e S. Sede. La stampa diede notorietà all'incontro, specie all'indirizzo che il porporato pronunciò, elogiando il duce per la «chiara visione della realtà delle cose», e per il proposito di fare in modo che la religione fosse «rispettata, onorata, praticata». ${ }^{25}$ Sulla scia della popolarità dell'Assisiate pro-

24 F. CHABOD, L'Italia contemporanea (1918-1948), Torino,1961. Sulla specifica natura del sistema allora inaugurato si vedano J. PETERSEN, Nachwort. Zum Stand der Faschismusdiskussion in Italien, in R.DE FELICE (ed), Der Faschismus. Ein Intervierw von Michael A. Ledeen, Stuttgart 1977, pp. 114-144, e più in generale, G.SCHULZ, Faschismus-nationalismus. Versionen und theoretische kontroversen 1922 1972, Frankfurt 1974.

25 Da vedere l'ampio servizio, corredato di fotografie dal titolo Cronache francescane (dal nostro inviato speciale [che si firmava Frate Lupo]), in "L'illustrazione Italiana", 10 ottobre 1926, pp.286-289. Si veda anche la cronaca su "La Civiltà Cattolica", 1926, IV, pp.175-176. A proposito dell'intervento di Merry del Val, si veda A.ZAMBARBIERI, Merry del Val, Rafael, in Dizionario Biografico degli Italiani, 72 (2009), p.743. Da consultare Ch.DUGGAN, Fascist Voices. An Intimated history of Mussolini's Italy, Oxford 2003, pp. 109 e seguenti. Per ulteriori approfondimenti: F.TORCHIANI, 4 ottobre 1926. San Francesco, il regime e il Centenario, in T.CALLIÓ, R.RUSCONI, San Francesco d'Italia. Santità e identità nazionale, Roma 2011, pp.76-99. Si vedano pure altri saggi raccolti nel medesimo e inoltre D.MENOZZI, "Il più italiano dei santi e il più santo degli italiani". La nazionalizzazione di San Francesco tra le due guerre, in Id.(ed), Cattolicesimo, nazione e nazionalismo, Pisa 2015, pp-87-109. 
cedette la predicazione largamente diffusa attraverso il nuovo mezzo, per molti versi rivoluzionario, della radiofonia. Il francescano Vittorino Facchinetti, detto "frate microfono", cominciò, proprio durante la celebrazione del ricordato centenario, le sue conversazioni religiose via etere, dove non mancarono cenni favorevoli al regime, specie nel prosieguo di tempo, ad esempio durante la guerra libica. ${ }^{26}$ Una visiva rappresentazione di convergenze nella circostanza si poté vedere durante quei giorni, sulla facciata della basilica di S. Maria in Aracoeli al Campidoglio nei pressi dell'antico convento dei frati minori: accanto al motto francescano Pax et Bonum, erano affiancati lo stemma di Roma e il fascio littorio. ${ }^{27}$ Vero è che nelle tangenze con la politica del momento, la religione possedeva, a causa di atavici retaggi, un ascendente senza dubbio superiore e una grande pervasività accolta nella vita quotidiana, dato l'alto tasso di frequenza alle consuetudini parrocchiali. ${ }^{28}$ Le persuasioni progressivamente irrobustite ai vertici e la nuova atmosfera creatasi nel paese, non furono comunque estranee, quantunque sia difficile stabilirne la precisa portata, al procedere dei negoziati fra il Vaticano e il Governo. Le relative fasi sono ampiamente studiate, così come la conclusione raggiunta in quel febbraio $1929 .{ }^{29}$

3. La carrellata sulle reazioni dei cattolici italiani, dopo l'annuncio del Trattato e del Concordato, pur condotta selezionando con una certa arbitrarietà e quindi con scontati limiti, offre una campionatura che, come già accennato, va dal consenso maggioritario a prese di distanza, quando non a ripulse, sebbene l'uno e le altre si connotino mediante diverse motivazioni e sfumature. Alcune voci sembrano particolarmente eloquenti.

26 S. AIROLDI, Gli esordi dell'apostolato via etere: le radio prediche di Vittorio Facchinetti (1926-1936), in "Società e storia", 2011, pp. 301-330.

27 S. ROGARI, Santa Sede e fascismo dall'Aventino ai Patti lateranensi, Bologna 1977, p.102.

28 S. COLARIZI, L'opinione degli italiani sotto il regime (1929-1943), RomaBari 1991, p.115.

29 Le disamine in proposito sono molteplici e dettagliate: tra le molte, soprattutto MARGIOTTA BROGLIO, Italia e S. Sede, pp. 180-257 e, indispensabile, R. DE FELICE, Mussolini il fascista. II. L'organizzazione dello Stato fascista 1923-1929, Torino 1968, pp. 101-114, 382-431; sempre utile A. MARTINI, Studi sulla Questione romana e la Conciliazione, Roma 1963. 
Palesò con solerzia la propria soddisfazione l'arcivescovo di Pisa Pietro Maffi, noto per la predilezione, fin da giovane, verso studi specie nel settore delle cosiddette scienze esatte e per la sua tendenza conciliatorista. Tra i candidati al papato nel Conclave del 1922, non nascose di mirare ad una rapida soluzione della questione romana. ${ }^{30}$ Sicché quando venne raggiunta vi dedicò il 17 febbraio del 1929 la lettera al clero dell'archidiocesi di Pisa il cui contenuto era compendiato già nell'introduzione: «Dopo quasi sessant'anni di angustie e di disagio, la riconciliazione tra la Chiesa e lo Stato, colla risoluzione ultima e definitiva della gravissima questione romana, si è avuta ed ora dunque concordia e pace! Esaudite così le preghiere fervide di mille e mille anime buone [...], compiuti i voti di quanti, per il bene di tutti sospiravano sul nostro cielo un ritorno di calma e di serenità, ed a noi ridata così la felicità di unificare in un palpito solo senza più contrasti i nostri voti di prosperità e di bene, per la Chiesa e per la Patria, per la santità del Padre e per la Maestà del Re [...]. Si commosse e sussultò all'atto tutto il mondo e l'Italia, anche sotto le raffiche nordiche che in quei dì la rivestivano di neve, tutta tripudiò e sussultò in un fremito, in un parossismo di esultanza, di letizia, di gaudio, che noi stessi che l'abbiamo vissuto, a stento lo potremo, quanto fu grande, ricordare». ${ }^{31}$ Intonazioni simili, quantunque su scale cromatiche non identiche, si udirono da molte parti. Buona eco ebbe la voce del barnabita Giovanni Semeria, molto popolare per le sue molteplici attività, dall'oratoria sacra ai volumi di cultura religiosa, dalla presenza al fronte - presso il Comando supremo - durante la guerra, alle iniziative filantropiche insieme a don Minozzi in favore degli orfani del conflitto e in genere del sud Italia. Sollecitamente consegnò un commento al "Corriere d'Italia," pubblicato proprio l'11 febbraio, giorno della firma dei Patti. Con enfasi li caratterizzava non come «una discesa a Canossa», ma piuttosto come «un'ascensione mistica al Colle Vaticano da parte della nuova Italia». A

30 F. ENGEL-JANOSI, Il Vaticano fra Fascismo e Nazismo, pp.83-84. Su di lui si veda il recente volume di G.CAVAGNINI, Per una più grande Italia: il cardinale Pietro Maffi e la prima guerra mondiale, Pisa 2015. Sempre interessante il precoce profilo di M.VAUSSARD, L'intelligence catholique au XXe siècle, Paris 1921, pp. 214-255. Va segnalato che nel 1924 apprezzò il Regio Decreto che stabiliva per ogni aula di scuola media l'esposizione del Crocifisso: Il Crocifisso ritorna. Lettera pastorale per la quaresima 1924, Torino 1924.

31 Card. PIETRO MAFFI, Conciliazione e Pace. Lettera al Clero dell'Archidiocesi di Pisa, Pisa 1929, pp.3 e 7. 
suo avviso si era pervenuti alla «logica conseguenza di una politica positiva» e, ciò che più importava al convincimento che «senza religione vera e profondamente rispettata da parte dei pubblici poteri un popolo non vive». In conclusione, occorreva «ricordare con rispetto e affetto due nomi che la data dell'11 febbraio consacra alla storia italiana ed ecclesiastica per sempre: Benito Mussolini e Pio XI». Le frasi andrebbero lette in controluce con le precedenti aspirazioni del religioso che già nel 1904, in un memoriale sull'abolizione del non expedit, preparato per il vescovo di Cremona Geremia Bonomelli da presentare a Pio X, si diceva persuaso che la patria andava difesa e insieme riconciliata con il papato dipinto a torto dai rivoluzionari come nemico, mentre al contrario sarebbe stato in grado ancora una volta di procurare la vera «salute d'Italia». ${ }^{32} \mathrm{E}$, avvicinandosi all'evento, aveva steso in un inedito manoscritto, alcune note sulla Conciliazione in marcia, da correlare ai suoi voti per una «cristianizzazione del fascismo». ${ }^{33} \mathrm{Da}$ rilevare come poi il barnabita si astenne da pubbliche esternazioni decisamente critiche verso il regime, mantenendo relazioni con personaggi politici e i relativi apparati. I suoi scopi erano volti ad ottenere sussidi frequentemente necessari per le proprie opere caritative. ${ }^{34}$ Sempre tra gli ecclesiastici Adriano Bernareggi, non dimentico delle discrete avances espresse all'inizio degli anni venti, manifestò vivo apprezzamento per l'intesa, nonché la stima verso Benito Mussolini, un uomo che, a suo dire e secondo il giudizio ripetuto tra il clero, aveva eliminate «tutte le grette concezioni di un liberalismo sorpassato». ${ }^{35}$

32 Il Memoriale si trova in Biblioteca Ambrosiana. Milano, O 32 inf., f. 11: da vedere in proposito C. MARCORA, Lettere di p. Giovanni Semeria a mons. Geremia Bonomelli, estratto da "Il bene" 196721 pp. Notizie del manoscritto nelle indicazioni delle fonti e bibliografia alla voce di A. GENTILI, Semeria Giovanni, in DSMCI II, pp. 596-602.

33 Si veda F. LOVISON, Dal "biennio rosso" all'avvento del fascismo. Appunti inediti di Padre Giovanni Semeria, in "Barnabiti studi", 27 (2010), pp.261-288.

34 In proposito son da vedere il ricordo e le spiegazioni di G. LEVI DELLA VIDA, Fantasmi ritrovati, Venezia 1966, pp.89-101. L'autore riporta lo stralcio di una conversazione avuta con il barnabita, che avrebbe giudicato la conciliazione «cosa buona» e liquidato, invece, il concordato come «cosa cattiva» (ivi, p. 105). Per quanto concerne i suoi rapporti con Mussolini è da vedere la documentazione in Archivio Centrale dello Stato, Presidenza Consiglio dei Ministri, Segreteria Particolare del Duce, Carteggio ordinario fasc. 512661.

35 A. BERNAREGGI, Iustitia et Pax osculatae sunt se, in "La scuola cattolica," 57/1, (1929), p. 92. 
In ambito gesuitico il p. Giuseppe Massaruti, uno tra i superiori del romano Istituto Massimo da cui passarono molti allievi diventati personalità della classe dirigente del paese, trasponeva la voce della vecchia Roma papalina, notoriamente ostile allo Stato italiano, su un'altra scala cromatica, riuscendo a sopire nostalgiche geremiadi, per salutare commosso quanto sancito dai Patti. Vedeva nella Conciliazione «il coronamento di un palpito antico», quello dei cattolici che «alle scuole imparavano ad amare il papa e l'Italia, a lamentarne il funesto dissidio e a desiderarne la pace». ${ }^{36}$ Il più prestigioso confratello p. Enrico Rosa, direttore della "Civiltà Cattolica", sul fascicolo della rivista uscito il 16 febbraio, pubblicava un articolo prima sottoposto alla revisione del papa. ${ }^{37}$ Il titolo, L'ora di Dio, espressione spesso ripetuta in quei mesi, conferiva un sigillo trascendente alla congiuntura ritenuta felice, poiché, secondo l'autore, i Patti costituivano «il riconoscimento pratico e solenne della religione cattolica apostolica romana come la sola religione dello Stato», a cui l'Italia era «tornata nella sua legislazione, nella sua educazione, nella sua vita domestica e civile privata e pubblica». ${ }^{38}$ Poco dopo sulla stessa rivista egli confessava di provenire da una «famiglia papalina», ma «buzzurra», che però poteva vantare, nella parentela piemontese, alcuni degli «inconsapevoli eroi», orgogliosi «di essere andati proprio essi a Roma per liberare il papa dai Francesi»; perciò con più convinto entusiasmo salutava un'altra liberazione, quella sancita dai Patti, appunto «allo scoccare dell'ora di Dio».39

Diversa la sensibilità che connotava gli altri ambienti. In alcuni si era passati dall'immediata spontanea contentezza ad una più meditata ponderazione. È il caso esemplato, per il nucleo dei cattolici liberalconciliatoristi, dalla cognata di Tommaso Gallarati Scotti, imparentata

36 In un articolo da "Il Massimo", periodico dell'Istituto (maggio 1929), citato da G.MARTINA, Storia della Compagnia di Gesù in Italia (1814-1983), Brescia 2003, p.259. Su Massaruti si ricavano notizie e una caratterizzazione autobiografica da una sua opera, curata con preziose note da Gabrio Lombardi: G. MASSARUTI, Da te consolato, con te consolatore, Milano 1975.

37 Ibid, p. 260.

38 L'ora di Dio, in "La Civiltà cattolica", 1929 I, p.293.

39 Lettera di Ugo Oietti e risposta del P. Enrico Rosa, in "La Civiltà cattolica," 1929 II, p.18 : l'intero articolo (pp. 4-18) costituiva la risposta ad una lettera in cui lo scrittore aveva espresso il timore che gli accordi preludessero ad un'invadenza culturale dei cattolici, pregiudizievole per la libertà dell'arte. 
con Antonio Fogazzaro, la quale non aveva trattenuto il giubilo all'annuncio dei Patti ma cambiò parere grazie a Benedetto Croce che ne smorzò l'esultanza, facendo leva sul paradosso che quella Conciliazione «la Chiesa aveva pervicacemente rifiutata ai grandi cattolici del Risorgimento ed aveva ora negoziata e conclusa con il miscredente, amorale, e beffardo capo della banda fascista»..$^{40} \mathrm{Il}$ contraccolpo fu negativamente e sconsolatamente avvertito, com'era da aspettarsi, dai Popolari. In verità anche qui a tutta prima Stefano Jacini jr, membro della tetrarchia che dal dicembre 1925 aveva retto il partito fino allo scioglimento voluto dal regime, si mostrò incline ad un certo attendismo. Legato a Gallarati Scotti, anche per l'antica solidarietà nella collaborazione alla rivista modernista "Il Rinnovamento," avrebbe potuto anche scorgere nei Patti il coronamento di aspirazioni conciliatoriste, ma il giudizio parzialmente positivo virò di fronte alle pretese egemoniche mussoliniane. ${ }^{41}$ Comunque, per la maggioranza dei seguaci del Partito Popolare, la notizia degli accordi ebbe l'effetto, secondo l'immagine di una lettera conservata nell'archivio sturziano, di «una valanga su una capannuccia di frasche» ${ }^{42}$ Rende efficacemente gli umori degli antifascisti una pennellata di Arturo Carlo Jemolo che riandando nel suo classico volume ai tempi e ai luoghi di cui fu testimone, ricorre al presente storico: «Chi penetrasse nei loro cenacoli, sentirebbe frasi un po' roboanti, che ricordano vecchi tempi: il Papa che inchioda il coperchio alla bara delle libertà degli italiani». ${ }^{43}$ Pur con intonazioni diverse, furono analoghi nella sostanza gli accenti di altri militanti nel partito di Sturzo. Appunto al leader, due giorni dopo la firma dei Patti, Francesco Luigi Ferrari si rivolse con un amaro sfogo: «se leggendo le notizie relative alla soluzione della questione romana, mi rammarico che proprio al fascismo sia stato concesso ciò che ai governi democratici si negava, apprendendo i principi generali del Concordato provo un senso di delusione, di disgusto, di pena, di paura. Il metodo della libertà

40 P. GADDA CONTI, "La famiglia italiana" a Milano, in A. PELLEGRINI (ed.), Tre cattolici liberali, Milano 1972, pp.327-328.

41 F. FONZI, Stefano Jacini Junior, ibid., p.253.

42 La citazione, con l'ipotesi sull'autore della lettera (forse Tarchiani), è tratta da G.ROSSINI, Per una storia dei Patti Lateranensi, in G. ROSSINI (ed.), Modernismo, Fascismo, Comunismo. Aspetti e figure della cultura e della politica dei cattolici nel '900, Bologna 1972, p.482.

43 JEMOLO, Chiesa e Stato, p. 469. 
ha dunque fatto sì completo fallimento che si deve ritornare alle vecchie forme di "clericalizzazione" degli istituti di diritto pubblico e privato [...]. Nessuna ripugnanza si prova in Vaticano a trattare con gli uomini le cui mani grondano di sangue umano, coi governanti che tengono in obbrobriosa schiavitù quaranta milioni di italiani, coi rappresentanti di una idea che pretende di sostituire al Cristo universale lo Stato Nazionale». ${ }^{44}$

Luigi Sturzo, rispondendogli, distingueva il Trattato dal Concordato. Sul primo il giudizio era positivo, poiché serviva a chiudere definitivamente la questione romana, ed era «un guadagno per la Chiesa che la Santa Sede abbia rinunziato a qualsiasi dominio territoriale a garanzia internazionale». Il secondo, andava considerato «più o meno», alla stregua di tutti i concordati, cioè «guadagni e perdite». Perciò non si doveva «combattere il fatto, né credere che il Papa abbia tradito gli interessi della morale religiosa». Piuttosto occorreva agire in modo che la Chiesa non si facesse avvolgere «dalle spire politiche del fascismo». ${ }^{45}$

Sulla medesima piattaforma di contrasto al movimento mussoliniano, ma seguendo un indirizzo del tutto personale, si mosse Giuseppe

44 Francesco Luigi Ferrari a don Sturzo, Lovanio 13 febbraio 1929, in ROSSINI, Per una storia dei Patti Lateranensi, p.498. Scritti che Ferrari pubblicò fuori d'Italia, sono editi a cura di M. C.Giuntella, in L. FERRARI, Scritti dell'esilio, I, L'Azione Cattolica e il Regime e altri saggi editi e inediti sui rapporti Chiesa Stato, Roma 1991. Su di lui son da consultare gli Atti del convegno nazionale di studi (Modena 27-28 maggio 1983), Francesco Luigi Ferrari a cinquant'anni dalla morte, Roma 1983.

45 Sturzo a Ferrari, Londra 16 febbraio 1929, in L. STURZO, Scritti inediti, 1924-1940, a cura di F. RIZZI, II, Roma 1975, p.223. Molto istruttiva la discussione protrattasi a lungo tra Sturzo e Gaetano Salvemini (quest'ultimo unitamente in alcune fasi anche a Giorgio La Piana) sulla portata sia dei Patti sia in genere dei rapporti Vaticano e Fascismo. Ancora negli anni Quaranta Sturzo sottolineava che «se un concordato presenta un colore politico, ciò non viene dalla Chiesa, ma dallo Stato contraente, che, purtroppo, ne usa a suo vantaggio». Al che Salvemini osservò che «le implicanze politiche favorevoli al regime fascista furono reali [...], note a Pio XI e accettate da lui come parte della trattativa». Si vedano i relativi testi in L. STURZO-G. SALVEMINI, Carteggio (1925-1957), a cura di G. Grasso, Soveria Mannelli 2009, pp. 128-130. Utilissime le puntualizzazioni del curatore nell'introduzione ai carteggi, specialmente pp. XXV-XXX. È da ricordare anche il giudizio che il fondatore del Partito Popolare espresse più tardi in L. STURZO, Chiesa e Stato. Studio sociologico-storico, II, Bologna 1959, pp. 174-178. In genere sulle reazioni dei Popolari si veda A. CANAVE$\mathrm{RO}$, I cattolici antifascisti italiani tra emigrazione ed esilio "in interiore," in L'émigration politique en Europe au XIXe et XX ${ }^{e}$ siècles, Rome 1991, pp. 344-370. 
Donati, già membro autorevole del Partito Popolare dove aveva ricoperto qualificati incarichi, tra cui quello di gerente responsabile del giornale "Il Popolo", un foglio che funse non solo da portavoce del partito ma anche, nella terza pagina, da collettore di contributi della cultura liberal-democratica, tra cui quelli di Croce, Gobetti, Salvatorelli, Dorso e Salvemini. Quest'ultimo in un appello agli elettori della circoscrizione Udine-Belluno, per le elezioni del 1919 l'aveva presentato come «non solamente un uomo nuovo, ma una coscienza nuova». ${ }^{46}$ Costretto all'esilio, anche per le sue battaglie contro il quadrumviro Emilio De Bono e Italo Balbo, continuò a manifestare il suo forte dissenso sia nei confronti della politica vaticana verso il regime sia ovviamente verso quest'ultimo. Ciò non gli impedì di emettere un giudizio parzialmente positivo a riguardo degli accordi lateranensi, perché a suo avviso con essi si era decisamente chiusa la questione romana e inaugurato il reciproco riconoscimento tra Stato italiano e Chiesa. Tale opinione, espressa soprattutto in articoli, firmati con il proprio nome sul quindicinale "Il Pungolo" gli attirò critiche da parte di altri fuoriusciti. E tuttavia, quando sullo stesso foglio scrisse sotto lo pseudonimo di Alessandro Di Severo, condusse una critica alla Conciliazione dall'angolatura dissimile, anche se per lui non alternativa a quella politica, di istanze atte a mettere in evidenza l'autonomia e la spontaneità del fenomeno religioso, nonché il relativo, inalienabile valore etico-sociale che ne scaturiva. Così un mese dopo la firma dei Patti, pubblicò sempre con lo pseudonimo, un saggio dove insisteva sui vantaggi dell'apporto di una religiosità che fosse in sintonia con i moderni risultati della critica storica (rivelatrice era la citazione di Loisy), suscettibile di svincolare l'adesione cristiana da ipoteche conservatrici e politiche, e di affermare il primato della mistica rispetto al diritto canonico e ad ogni alleanza col potere civile. Per lui, dal connubio tra l'uno e l'altra era sorto il fascismo: dunque occorreva superarli perché nel suo giudizio (qui però parla come Di Severo) sembravano funzionali al fascismo, promananti da tendenze radicate nella cultura e nelle prassi italiane su cui si era innestato il movimento mussoliniano. Cioè il fascismo germinava da un sostrato alieno da un' autentica religiosità: «L'attuale Italia areligiosa così le sue parole - è figlia della precedente Italia areligiosa ma clericale,

46 Si veda STURZO- SALVEMINI, Carteggio. Introduzione, pp. XI-XII; per i rapporti Donati- Salvemini, cenno a p.VIII. 
anche quand'era anticlericale». Sicché il problema della libertà, dopo gli accordi del Laterano, investiva non solo i rapporti di carattere politico bensì «il fondo stesso del fatto religioso, come esigenza razionale di religiosità e come esigenza sociale di religione» dal momento che nel «cristianesimo romano» prevaleva il convincimento che l'autorità effettiva risiedesse non tanto «nell'ispirazione, nella rivelazione, nella tradizione, quanto nella volontà imperiale e direttiva del papato», essendo i Patti «l'applicazione letterale di codesta dottrina», etichettabile come clericalismo. In altri termini il problema del rapporto con il fascismo diventava anche una questione di riforma ecclesiale. ${ }^{47}$

Va constatato che, nelle file dei Popolari, non mancò, chi sulla direttrice di don Sturzo riuscì a metabolizzare in modo realistico il pur evidente sconcerto. Quantunque non dissimulasse il proprio disappunto, Alcide De Gasperi tentava un approccio sulla linea della politique d'abord a lui più congeniale. Egli, da poco scontata una condanna per espatrio clandestino, ${ }^{48}$ rimanendo a Roma agli inizi di quel febbraio, era in trepida attesa di una positiva risposta alla sua domanda di impiego nella Biblioteca Apostolica Vaticana. Non tralasciò di fornire informazioni agli amici trentini. Rese partecipe un amico sacerdote di una specie di reportage su quanto accaduto il giorno dopo la firma dei Patti, premettendo una confidenza sul malanno che l'aveva colpito in quella gelida stagione: «L'influenza mi costringe ancora in camera, e così vedo dalla finestra la fiumana di cattolici e di italiani che tornano da S. Pietro». Erano duecentomila le persone, secondo il colpo d'occhio della moglie Francesca, che avevano sostato nella grande piazza sotto la pioggia. Lo spettacolo non distoglieva Alcide dai problemi personali immediati, né da quelli a scadenza più dilatata del suo impegno pubbli-

47 A. DI SEVERO, Il problema religioso italiano e gli accordi fascisti-vaticani, in “ Il Pungolo", 15 marzo 1929, pp.50-54. Un'acuta disanima del pensiero di Donati su questi temi e in genere sulle posizioni dei popolari è quella di F. TRANIELLO, Fascismo e storia d'Italia nei popolari in esilio, in Id. Città dell'uomo. Cattolici, partito e Stato nella storia d'Italia, Bologna,1990 pp. 139-168; su Donati specialmente pp. 141147; 155-168. Per riferimenti biobigliografici su questo interessante personaggio che morì in esilio a Parigi non ancora quarantenne, nel 1931, è da vedere la voce dedicatagli da G. IGNESTI, in DSMCI, 2, pp. 181-190.

48 M.R. CATTI DE GASPERI, De Gasperi uomo solo, Milano 1964, pp.114116, e G. ROSSINI, Il movimento cattolico nel periodo fascista, Roma 1966, pp. 225-243 (e i documenti alle pp. 245-265). 
co. Con una vena d'ironia riferiva sulle incertezze per la sua assunzione presso la prestigiosa istituzione, assicurando di aver informato gli uomini di Curia circa il desiderio di non voler creare neppure «il minimo imbarazzo all'autorità religiosa». E postillava: «Ora vedremo: le vie della Provvidenza sono misteriose. Per sciogliere la questione romana ha convertito un socialista; forse per sfamare me commoverà, più che $i$ cattolici, gli ebrei». Lo interessavano nelle prospettive del suo agire politico le opinioni che, a botta calda, circolavano tra gli ecclesiastici e i fedeli del laicato: «I cattolici di qui sono variamente commossi, i vecchi popolari sono furibondi, perché temono una compromissione col regime e perché nel trattato si discorre di miliardi; i temporalisti più accesi, compresi i gesuiti, portano intorno una faccia trionfale, come se invece delle idee del Calabiana, del Tosti, del Bonomelli, della Lega Lombarda, avessero prevalso quelle della "Civiltà" del P. Zocchi e dell'"Osservatore cattolico" [...]. Quindi contenti i clerico-papalini, contenti i fascisti, contenti i massoni, Mussolini è trionfante e Briand si dice lietissimo». ${ }^{49}$ Lasciata la cronaca, l'uomo politico passava ad una valutazione più comprensiva, e con il senno di poi più comprensibile: «Credo che anche oggi, di fronte a Mussolini che picchiava forte alla porta di bronzo, il Papa non poteva non aprire e, una volta avviata la conversazione e trovato il terreno d'accordo, il suo alto senso di responsabilità, l'entità stessa della questione, lo portavano a conchiudere. La conclusione è, vista oggi in Italia, un successo del regime, ma vista nella storia e nel mondo è una liberazione per la Chiesa e una fortuna per la Nazione Italiana. Non si poteva esitare e credo che avrebbe firmato, fosse stato Papa, anche D. Sturzo. Per me l'essenza è che la $\mathrm{S}$. Sede è uscita dal vicolo chiuso delle proteste (vicolo che per sua natura non può essere eterno) ed ha liquidato la questione temporale senza i pericoli e gli aggravi del territorio e senza la complicazione di formule internazionali». E, ritornando ancora al piano personale, ma non solo, confessava con sorridente polemica di provare «la consolazione di essere gli ultimi sacrificati. In verità a noi la libertà arriva quando non ne possiamo usare, e siamo diventati maggiorenni quando ci hanno portato via il

49 Da notare che il Presidente francese, a suo tempo protagonista della Loi de séparation de l'Eglise et de l'Etat (1905: si veda J-M. MAYEUR, Paris-Mesnil-Ivry 1966), aveva inviato a Pio XI, l'11febbraio 1929, giorno della firma dei Patti, un telegramma di rallegramenti. 
patrimonio; ed è certo che nascere disgraziati è una disgrazia; ma come figli della Chiesa dobbiamo gioire. Certo ne guadagna il regime, si rinforza cioè la dittatura che dovrebbe pur essere un sistema transitorio ma questa considerazione non poteva essere decisiva né per la conclusione né a noi per giudicare. Il pericolo piuttosto è nella politica concordataria. Ne verrà una compromissione della Chiesa come in Spagna

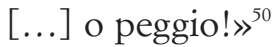

Dai palazzi vaticani, impegnato nelle incombenze della Segreteria di Stato, Giovanni Battista Montini soppesava gli avvenimenti, per lui non inaspettati data la sua carica, in modalità articolate. Ne scrisse il 18 febbraio alla famiglia che viveva a Brescia. Innanzitutto si premurava di attenuare i toni trionfalistici accesi nella circostanza. E proseguiva con la nota meteorologica, per sconfinare in una disincantata cronaca: «Giornate fredde queste anche per l'entusiasmo che si attendeva al grande avvenimento: il popolo, ormai lontano dalla cosa pubblica - ciò che non è del tutto male - ed avvezzo a rimanere indifferente nell'anima allo strepito artificiale dei giornali - ciò che è peggio, non per l'indifferenza, ma per l'artificio forzato ed inane - osserva senza commenti e tira via». ${ }^{51}$

Sempre di Montini un bilancio meditato e volto al futuro si legge in un commento che egli pubblicò sul periodico della Federazione Universitaria dei Cattolici Italiani di cui era assistente ecclesiastico nazionale. Dopo aver constatato come la firma dei Patti avesse «recato un certo grande piacere per la fine di un'annosa e dolorosa questione», ma insieme prodotto «qualche turbamento nell'orientamento pratico dell'associazione», si chiedeva, secondo il suo consueto stile interpellativo spesso ritmato sull'antitesi: $:^{52}$ «Possiamo e dobbiamo ancora continuare su la via intrapresa? Se l'atteggiamento nobilmente polemico che ha tante volte coinciso con gli scopi più alti del nostro apostolato, risul-

50 De Gasperi a don Simone Weber, Roma 12 febbraio 1929, in A. DE GASPERI, Lettere sul Concordato, con saggi di M.R. CATTI DE GASPERI, e di G. MARTINA, Brescia 1970, pp.59-66.

51 La lettera reca la data Roma 18 febbraio 1929, in G. B. MONTINI, Lettere ai familiari (1919-1943), II, Brescia-Roma 1986, pp. 588-589.

52 Interessanti osservazioni al riguardo in F. FINOTTI, Critica stilistica e linguaggio religioso in Giovanni Battista Montini, Brescia-Roma 1989, spec. pp. 68-97; qualche esempio anche in A. ZAMBARBIERI, Dissonanze e accordi. Note su Paolo VI e $i$ vescovi italiani al Vaticano II, in E. ROSANNA (ed.), Il Concilio e Paolo VI a cinquant'anni dal Vaticano II, Brescia-Roma 2016, pp.317-318, e 392. 
tasse ora e poi (...magari!) anacronistico abbiamo ancora scopo sufficiente per vivere? Se eravamo milizia, ora con questa pace che s'è fatta, non cadono per ciò stesso dalle nostre mani le armi? [...].La F.U.C.I. non avrebbe più nulla da fare perché non ha più da combattere? Non è poi vero che la milizia dell'idea cristiana era la sola ragione della nostra esistenza? E l'opera formativa dei giovani? E la diffusione pacifica di quell'idea? E la pratica integra e vivente di essa? Di più: è poi vero che non ci sia più campo di battaglia per l'idea cristiana? Ha essa trionfato dovunque? No so altrove: certo nell'università questa idea è tutt'altro, pure adesso, che vittoriosa! $\mathrm{O}$ che forse la combattività giovanile va solo considerata sotto un aspetto di baruffa goliardica, piuttosto che di affermazione ideale e morale?». Agli interrogativi, la risposta: «Tutta la ricchezza morale, tutta la spinta ideale, tutta la bellezza programmatica del nostro movimento non vengono meno adesso per nessuna ragione. Anzi la logica delle cose vorrebbe che fosse maturata per essa l'ora propizia». Occorreva dunque lasciarsi alle spalle il passato e cambiare registro, quando «molti che si dicevano cattolici erano tali per il solo fatto che si volevano differenziare dagli altri e non badavano con questo a due importantissime esigenze della loro professione di cattolici: la pienezza e l'integrità della vita cristiana, della formazione cristiana, da un lato, e la necessità, reclamata dalla natura salvatrice e diffusiva della verità, di penetrare e di espandersi fuori della barriera chiusa della propria trincea difensiva. Ora tutto dà a credere che nella vita italiana non debba più sussistere una voluta e qualificata perversità anticlericale, e che dovunque possa rigermogliare la gloriosa e genuina tradizione cattolica. Dio lo voglia! Questa situazione viene a modificare ma non a togliere, o ad attenuare la forma del nostro agire». Il sacerdote bresciano si rifaceva a persuasioni già maturate e a precedenti consigli impartiti ai giovani universitari. Sulla scia della spiritualità e dell'impegno culturale del cenacolo bresciano della Pace, optava per un'opera educativa basata sull'approfondimento di testi cristiani specialmente liturgici e biblici in modo che dai gruppi elitari tutto ciò rifluisse sulla compagine dei fedeli. ${ }^{53}$ Disegnava cioè, sulla mappa della cattolicità italiana, un iti-

53 G.B. MONTINI, Scritti fucini, a cura di M. MARCOCCHI, Roma 2004, pp.278-279. Per l'indirizzo diverso (e/o complementare) seguito da Agostino Gemelli con l'Università Cattolica, si veda R.MORO, La formazione della classe dirigente cattolica (1929-1937), Bologna 1979, pp.132-139, e per il complessivo atteggiamento del 
nerario dalle caratteristiche sufficientemente distinte, rispetto a tragitti seguiti da altri. Lo sfondo rimaneva quello di un'approvazione almeno tacita dell'accordo Stato-Chiesa, ma anche di un distacco dalle immediate urgenze politiche e comunque da una certa svalutazione delle intese di vertice, certo non sovrapponibile a quel "tirare avanti" avvertito tra le folle. Preminente per lui era l'obiettivo della formazione religiosa e culturale di cui doveva equipaggiarsi una futura classe dirigente italiana, attrezzata a istruire e a dirigere i ceti popolari.

La controllata prosa montiniana, tenendo conto dei giovani universitari cui era diretta, denota la capacità di cogliere i contingenti risvolti operativi, da discernere entro una complicata transizione. Vi si evidenzia la distanza da alcune riserve critiche avanzate negli ambienti vaticani, anzi all'interno del collegio cardinalizio. Le disapprovazioni, più $\mathrm{o}$ meno tacite, nascevano da orientamenti che auspicavano una confessionalizzazione dello Stato più netta di quella appena conseguita. Un singolare campionario al riguardo si può scorrere grazie ai rapporti degli informatori italiani a contatto con il Vaticano. Basti leggere la tagliente battuta di Merry del Val, il quale nel 1926, come si è visto, avrebbe implicitamente dato un discreto impulso ai lavori preparatori dei Patti. Commentando la frase di Pio XI, secondo cui per arrivare al Concordato sarebbe stata necessaria l'esperienza di un alpinista (da ricordare che papa Ratti era stato scalatore in gioventù), aveva acidamente commentato: «Si vede bene che il Concordato è stato fatto da un alpinista [...]. Giustissimo, è un concordato fatto con i piedi». ${ }^{54}$

Una panoramica generale, se si vuole superficiale, sugli umori dell'opinione pubblica è attingibile da un'inchiesta della Polizia. Essa atte-

futuro Paolo VI in questi anni una puntuale ricognizione è effettuata dallo stesso MORO, Giovanni Battista Montini e il fascismo, pp.41-65; si veda anche A. GIOVAGNOLI, Le premesse della ricostruzione. Tradizione e modernità nella classe dirigente cattolica del dopoguerra, Milano 1982, pp. 201-293. Da ricordare anche la sua attività di assistente al Circolo Universitario Romano, su cui A. RICCARDI, Roma città sacra, Milano 1979, pp.74-79.

54 M. CASELLA, Cesare Maria De Vecchi di Val Cismon. Primo ambasciatore d'Italia in Vaticano (giugno 1929-luglio 1935), in "Storia e Diplomazia", 2 (2009), pp.11-28; a pp.11-28: si riportano le reazioni di alcuni porporati, quello di Merry del Val a p.11. Commenti cardinalizi anche in CARLO M. FIORENTINO, All'ombra di Pietro. La Chiesa Cattolica e lo spionaggio fascista in Vaticano 1929 - 1939, Firenze 1999. 
sta un sostanziale assenso agli avvenuti accordi pattizi e una non avversione e frequentemente un favore, verso l'assetto politico ormai consolidato. Indicative, tra le molte, le relazioni riguardanti le province di Trento e di Vicenza. Per la prima, l'impressione era che il clero, dopo aver dimostrato una certa ostilità verso il regime, una volta firmati i Patti aveva assunto «un atteggiamento apertamente favorevole». Sostanzialmente identico quanto riferito per la seconda: «il movimento cattolico non desta più alcuna apprensione, perché esso, dopo l'avvenuto accordo con la Chiesa, va incontro al fascismo con manifesto spirito di simpatia». ${ }^{55}$ Qualche distinguo faceva capolino, ad esempio, per Udine: «Il clero nella quasi totalità, si addimostra abbastanza sereno ed ossequiente alle Autorità dello Stato. In alcuni sacerdoti permane però un certo spirito di resistenza verso le iniziative e le istituzioni di carattere fascista». ${ }^{56}$

Si potrebbe continuare nel reperimento di opinioni riscontrabili tra i cattolici, moltissime compiaciute ma qua e là pure critiche, in una campionatura difficile da imbrigliare. Coglieva infatti nel segno la battuta di una lettera che don Giuseppe De Luca indirizzerà nel 1931 due anni dopo a Prezzolini: «ciò che è i cattolici, ella sa che bisogna distinguere. In Italia, i cattolici ce n'è almeno 12 categorie». ${ }^{57}$ Nell'archivio Salvemini la copia di una lettera, probabilmente di Donati, dipinge pittorescamente alcuni ritratti di cattolici impegnati nell'arengo civile: «La barca di Pietro, purtroppo è come l'arca di Noè, piena di animali politici d'ogni specie, compresi quelli che non vanno d'accordo e si mangiano tra loro volentieri e facilmente». ${ }^{58}$ Un allineamento massiccio ma ambiguo di quasi tutte le categorie, si ebbe al Plebiscito del 24 marzo 1929. Come è noto, sciolto il parlamento, la nuova legge elettorale prevedeva un voto, positivo o negativo, a una lista di 400 candidati, prodotta su compilazione finale del

55 Situazione della Pubblica Sicurezza dal lato politico nelle Province del Regno, in Archivio Centrale dello Stato, Ministero Interni, Direzione Generale Pubblica Sicurezza, Divisione Affari Generali e Riservati (1920 -1945), Cat. 1, b. 94 (trimestre 1 gennaio -31 marzo 1929), da P. SCOPPOLA, La Chiesa e il fascismo. Documenti e interpretazioni, Bari 1971, pp. 242-243.

56 Ibid., p. 250 (trimestre dal 1 ottobre al 31 dicembre 1929).

57 Lettera del 23 marzo 1931, in G. DE LUCA - G. PREZZOLINI, Carteggio 1925-1962, a cura di G. Prezzolini, Roma 1975, p.83.

58 Il documento si legge nell'Introduzione a STURZO-SALVEMINI, Carteggio, pp. XV-XX. 
Gran Consiglio Fascista. Il 13 marzo la Giunta Centrale dell'Azione Cattolica Italiana richiamò i fedeli al «dovere di concorrere con il loro voto alla formazione della nuova Assemblea legislativa destinata a sancire e ad attuare le importantissime Convenzioni del Laterano, convinta che il perfetto adempimento di esse sarà uno dei contributi più necessari e più efficaci per l'auspicata prosperità e grandezza della Nazione»».59 Tre giorni prima, in un discorso a Milano, il presidente dell'organizzazione Luigi Colombo si era schierato per il dovere di dare un voto favorevole, spiegandone il valore: «Significherà approvazione a quanto l'on. Mussolini sottoscrisse, in forza delle regie credenziali; significherà riconoscenza verso il Capo dello Stato e il suo Governo; mandato impegnativo al Parlamento per la ratifica; mandato altresì di approvare tutte le leggi che saranno necessarie per l'integrale e volonterosa applicazione del Concordato». ${ }^{60}$ In due articoli pubblicati sull'"Avvenire d'Italia" il 23 e 24 marzo Enrico Rosa aveva precisato l'indole del voto che doveva rivestire « pure finalità religiose, destinato perciò a restare al di fuori e al di sopra di tutti i partiti». ${ }^{61}$ Da queste autorevoli premesse, non poteva che derivarne una pressoché unanime partecipazione dei cattolici, beninteso tenuto conto del diritto di voto riservato solo ai maschi maggiorenni. Sicché il 24 marzo si recò ai seggi l'89,46\% degli aventi diritto, per lo scontato successo dei suffragi positivi pari al $98,34 \%{ }^{62}$

4. Occorre ribadire come il responso plebiscitario venisse ottenuto non solo mediante i ricordati appelli ai cattolici, ma sull'onda di un intenso battage pubblicitario, lanciato pure tramite grandi apparati iconografici e l'utilizzo di artifici in grado di impedire la segretezza e la libertà dell'espressione elettorale. ${ }^{63}$ Interessante risulta peraltro l'esame condotto in sede storica sui risultati in specifiche zone, che, in pochi ma significativi

59 Così sull"'Osservatore Romano" del 17 marzo 1929, in P. SCOPPOLA, Chiesa e Stato nella storia d'Italia, Bari 1967, p. 624.

60 Cit. in PERTICI, Chiesa e Stato, pp. 167-168.

61 Cit. in L. SALVATORELLI - G. MIRA, Storia d'Italia nel periodo fascista, Torino 1956, pp. 461-462.

62 Istituto Centrale di Statistica del Regno d'Italia, Statistica delle elezioni generali politiche per la XXVIII legislatura (24 marzo 1929, anno VII), Roma 1930: le percentuali complessive alle pp. 47 e 56.

63 Basti citare lo studio di E. FIMIANI, "Raggiungi il Cento per Cento!". 
casi, presentano alcuni scostamenti dal trend generale. ${ }^{64}$ Comunque l'imponente risultato, nel rimbalzo delle enfasi nella comunicazione giornalistica, sembrava attestare una massiccia adesione popolare alla stipula dei Patti. Si trattava del decollo di una trionfale "luna di miele", come si suol designare il periodo successivo a raggiunte alleanze tra personaggi e partiti, nella fattispecie tra le autorità statuali e le ecclesiastiche? E inoltre, al di là dei ricordati dissensi nella composita geografia del cattolicesimo, si poteva in qualche misura ritenere "provvidenziale" quanto stipulato tra il Papa e Mussolini? L'aggettivo era indotto dal giudizio dello stesso pontefice, spesso sintetizzato dall'espressione vulgata "uomo della provvidenza", in cui il genitivo di elogio qualificava il capo del fascismo. Più esattamente l'espressione di papa Ratti suonò, in un discorso del 13 febbraio 1929, «un uomo come quello che la Provvidenza Ci ha fatto incontrare»: l'accento cadeva dunque sul protagonismo di un'azione trascendente, né sembra casuale che il papa parlando proprio nel giorno e forse addirittura nell'ora in cui i Patti vennero firmati, avesse usato il medesimo lemma. L'interrogativo da lui enunciato «Che sarà domani?», confessava la fiducia verso un futuro ancora impregiudicato, eppur consegnato all'assicurazione di un disegno superiore ritenuto affidabile: «qualunque cosa sia per essere il cenno della Divina Provvidenza lo seguiremo fidenti sempre in qualunque direzione chiami».65 Tale attitudine si riscontra fre-

Mobilitazione, adesione, coercizione nei plebisciti fascisti (1929-1934), in P.L. BALLINIM. RIDOLFI (edd.), Storia delle campagne elettorali in Italia, Milano 2002, pp. 168 . 192, e in generale sulla strategia dei plebisciti, ID., Per una storia delle teorie e delle pratiche plebiscitarie nell'Europa moderna e contemporanea, in "Annali dell'Istituto storico italo-germanico in Trento", 21 (1995), pp.267-333. Si veda anche A.AQUARONE, L'organizzazione dello Stato totalitario, Torino 1978, pp. 151-158.

64 Si veda ad es. P. DAL LAGO, Verso il regime totalitario. Il plebiscito fascista del 1929, Padova 1999.

65 Pio XI tenne il discorso dell'11 febbraio ai parroci e ai quaresimalisti di Roma (in Parole pontificie sugli accordi del Laterano, Roma $1929^{2}$ pp.5 -19) e in quello del 13 febbraio ai professori e agli studenti dell'Università Cattolica di Milano (ibid. pp. 25-32) aveva asserito: «Forse ci voleva anche un uomo come quello che la Provvidenza $\mathrm{Ci}$ ha fatto incontrare; un uomo che non avesse le preoccupazioni della scuola liberale, per gli uomini della quale tutte quelle leggi, tutti quegli ordinamenti, o piuttosto disordinamenti, tutte quelle leggi, diciamo, e tutti quei regolamenti erano altrettanti feticci $\mathrm{e}$, proprio come i feticci tanto più intangibili e venerandi quanto più brutti e deformi». Da notare nei due discorsi l'andamento colloquiale adottato dal pontefice con scontate ripetizioni, ma anche la sottolineatura del protagonismo della "Provvidenza" per mostrare in modo nitido che l'uomo politico era stato uno strumento di essa. 
quentemente negli uomini di Chiesa e, per fare un esempio attinente alla tematica della Conciliazione, comparirà anche nel discorso tenuto in Campidoglio da Montini, diventato arcivescovo di Milano, alla vigilia del Concilio Vaticano II. Nella sua valutazione la caduta del potere temporale era da connettere ad una disposizione della Provvidenza che aveva portato ad esiti molto favorevoli. ${ }^{66}$ Riguardo a Pio XI, la sua attitudine risultava sufficientemente articolata, come risalta in un colloquio che egli ebbe in data 25 marzo, il giorno successivo al voto plebiscitario, con Stefano Jacini jr. Il pontefice ammetteva di non aver potuto eludere la coazione di circostanze forse irripetibili: «come sfuggire alle responsabilità di non aver fatto quanto si poteva?». Certo rimaneva l'obiezione, fra l'altro avanzata dall'interlocutore, sul fatto di aver patteggiato con la Rivoluzione. Al che il papa rispose con il ricorso ad un'immagine per dir così didascalica asserendo che non «bisogna cessare di respirare perché in una stanza l'aria è inquinata» mentre al rilievo riguardante il Trattato, cioè la soluzione territoriale a preferenza della scelta di garanzie internazionali, ribatteva che queste non erano mai state nell'ottica della S. Sede. Alle riserve avanzate sul Concordato che Stefano ripresentava, in linea con le idee dell'omonimo nonno, convinto separatista, Pio XI aveva pronta la replica: «l'uomo è uno, non si può dividere in lui l'uomo civile dall'uomo religioso: quindi distinzione non separazione». ${ }^{67}$

"La Civiltà Cattolica", per la penna del direttore Enrico Rosa, arginava un'interpretazione estensiva dell'appoggio dei cattolici al regi-

66 G.B. MONTINI (Arcivescovo di Milano), Discorsi e scritti milanesi (19541963), III, Brescia-Roma 1997, pp. 5352-5353. Da rilevare come anche Angelo Giuseppe Roncalli, allora patriarca di Venezia, accennerà nel $25^{\circ}$ anniversario dei Patti a «quell'uomo che la Provvidenza fece incontrare il Santo Padre Pio XI», aggiungendo che la Provvidenza stessa «nella realizzazione dei suoi disegni suole scegliere i vasi più acconci all'uopo, e ad opera compiuta li spezza, come se non fossero stati preparati che per questo. Rispettiamo anche i pezzi del vaso infranto, e rendiamo utili per noi gli insegnamenti che di là ci provengono». A.G. RONCALLI, Scritti e discorsi, I, Roma 1959, p.167 (Ringrazio per la segnalazione l'amico prof. Michele Madonna). Annota acutamente, per un altro contesto e un altro pontefice, Pio XII, le implicazioni di riferimenti a prospettive di stampo religioso e latu sensu teologico, G. MICCOLI, I dilemmi e $i$ silenzi di Pio XII, Milano 2000, pp. 406-413. Per una prospettiva storico-teologica sul concetto di Provvidenza si può vedere W. KERN, Provvidenza di Dio e conservazione del mondo, in J. FREINER-M. LÖHRER, Mysterium salutis, IV/2, Brescia 1970, pp. 172-192.

67 FONZI, Stefano Jacini Junior, pp. 253-254. 
me. Il suffragio elettorale non equivaleva all'approvazione in toto del regime. Anzi: «allo spirito e alle dottrine del cristianesimo ripugnano diametralmente le forme e concezioni dello Stato che molti vantano ai nostri giorni col titolo, più o meno abusivo di Stato fascista». ${ }^{68}$ Se le precisazioni di Rosa erano ben enunciate, tuttavia i dadi erano orami inesorabilmente tratti e la china dei distinguo non avrebbe arrestato l'irrobustirsi della dittatura. Peraltro alcune divergenze, già in nuce nelle accennate riserve, ebbero un pubblico innesco soprattutto durante la fase delle ratifiche parlamentari degli accordi pattizi. Prima alla Camera dei Deputati e poi al Senato, furono soprattutto i noti discorsi mussoliniani a risvegliare mai sopite discrepanze. ${ }^{69} \mathrm{Il}$ capo del fascismo, parlando in data 13 maggio a Montecitorio, aveva perentoriamente dichiarato che ormai in Italia esistevano «due sovranità ben distinte, ben differenziate, perfettamente e reciprocamente riconosciute». Ma la Chiesa non si poteva ritenere né "sovrana" e nemmeno "libera", poiché restava sottoposta «nelle sue istituzioni e nei suoi uomini» sia alle leggi generali dello Stato sia alle clausole speciali del Concordato. E dunque «Stato Sovrano nel Regno d'Italia; Chiesa Cattolica, con certe preminenze lealmente e volontariamente riconosciute; libera ammissione di altri culti». Imprescindibile restava il controllo dello Stato sull'istruzione e sull'educazione, quantunque l'Italia possedesse «il privilegio singolare [...] di essere l'unica nazione europea [...], sede di una religione universale». Ma a proposito del cristianesimo aggiungeva una glossa importante: nato in Palestina era però «diventato cattolico a Roma»: se qui non fosse approdato avrebbe seguito la sorte «degli Esseni e Terapeuti», estinguendosi «senza lasciare traccia di sé».70 Tali affermazioni ottennero l'unanime plauso fascista, mentre perplessità, sorpresa e anche sconcerto causarono fra i cattolici, a cominciare da Pio XI. Non accettabile per loro risultava il ridimensionamento dell'originalità, dell'influenza e delle radici storiche della Chiesa, così come della sua rilevanza istituzionale. Decisamente inaccettabile, la volontà di ridurre gli spazi assegnati ai compiti e agli istituti educativi degli enti ecclesiastici.

La rotta di collisione venne più chiaramente tracciata in seguito al

68 E. ROSA, La ristaurazione cristiana el" "unità della patria degli spiriti", in "La Civiltà Cattolica”, 1929, II, pp.193-204, citazione alle pp. 199-200, e 202.

69 Analitiche disamine in PERTICI, Chiesa e Stato, pp. 189-199 e pp. 215-219.

70 Testo in SCOPPOLA, Chiesa e Stato, pp.636-638. 
discorso mussoliniano del 25 maggio in Senato. Il senatore Benedetto Croce, annunciando il voto contrario proprio e quello di pochi colleghi, aveva effettuato una stringente analisi critica, specie delle norme concordatarie, per concludere che, se l'insieme era da giudicare dal punto di vista politico secondo il «trito detto che Parigi val bene una messa», egli si collocava sulla sponda di coloro «pei quali ascoltare o no una messa è cosa che vale infinitamente più di Parigi, perché affare di coscienza». ${ }^{71}$ Rimarchevole la lettera in cui Stefano Jacini jr. manifestava al filosofo la propria «ammirazione per lo spirito alto, pacato e sereno» di quell'intervento e in specie, soggiungeva, «per quella frase su Parigi e la messa, che avrei voluto sentir ripetere da altri, perché è davvero degna di un "uomo di Chiesa" nel senso più alto e più puro della parola». ${ }^{72}$ Perentoria in senato la replica di Mussolini a don Benedetto, e sprezzante quando lo tacciava di essere un «imboscato della storia», perché incapace di comprendere i progressi dell'Italia fascista, cioè di «una storia in fieri». ${ }^{73}$ Il duce ritornava sul tema dell'educazione come compito spettante allo Stato e sul decisivo contributo della civiltà romana per lo sviluppo del cristianesimo. In proposito si inoltrava, sorprendentemente, nella storiografia delle origini cristiane, citando le opere dei cattolici Pierre Batiffol (Battifolle nel testo) e Louis Duchesne, per evidenziare il ruolo dell'apostolo Paolo nel diffondere l'annuncio cristiano oltre il giudaismo, grazie soprattutto alla sua venuta a Roma dove il messaggio evangelico avrebbe trovato la propulsione universalistica. Il topos del decisivo influsso dell'Impero e della civiltà romana, prevalenti su ogni altro fattore nella configurazione e nell'affermazione del cristianesimo, faceva parte di una retorica fascista spesso reiterata. Su tale scia quei testi eruditi, pur menzionati al volo, davano l'ansa ad una puntata polemica del duce verso gli studiosi cattolici italiani: «debbo citare i francesi perché da qualche tempo in qua il cattolicesimo italiano non è fecondo, la produzione intellettuale in questa materia è altrove». ${ }^{74}$ In definitiva per Mussolini l'Italia fascista doveva essere stimata quale

71 Testo riprodotto in PERTICI, Chiesa e Stato, pp. 665-668. Per questo intervento, si veda D. MENOZZI, Croce e il Concordato del 1929. "Parigi non vale una messa", in M. CILIBERTO (ed.), Croce e Gentile. La cultura italiana e l'Europa, Roma 2016, pp. 573-580.

72 FONZI, Stefano Jacini Junior,pp.255.

73 PERTICI, Chiesa e Stato, p. 217, in nota con di riferimenti bibliografici.

74 Ibid. pp. 214-215. 
degna erede di Roma antica mentre il cattolicesimo aveva nella città eterna la sua capitale, costituendo così un elemento di raccordo tra l'Impero e il Medioevo. Le parole, che riproponevano un leit-motiv spesso ripetuto ${ }^{75}$ provocarono un'energica ripulsa da parte del papa, che ne scrisse a Gasparri in un chirografo datato 30 maggio, festa del Corpus Domini. Vi rivendicava la superiorità del cristianesimo e nella fattispecie l'anteriorità ideale e cronologica della sua irradiazione universalistica rispetto all'Impero, fatti, per il pontefice, da collegare a un volontà divina: «distinguere $[. .$.$] tra affermazione storica e affermazio-$ ne dottrinale sarebbe in casu del peggiore e più condannevole modernismo; il mandato divino alle genti universe è anteriore alla chiamata di San Paolo; anteriore di diritto e di fatto agli inizi primi della Chiesa e della predicazione apostolica; questa per opera degli apostoli e degli uomini apostolici è ben presto più vasta dell'Impero romano». E per transenna segnalava come il libro di Duchesne fosse stato messo all'Indice nel 1912 e il giudizio sulla scarsa incidenza della produzione intellettuale dei cattolici italiani dovesse venir ridimensionato perché troppo sommario. Per le prerogative pontificie, riaffermato «il carattere sacro della città di Roma, la città episcopale del successore di San Pietro», proseguiva dichiarando che nel papa era realizzata la pienezza della sovranità della Chiesa Cattolica, da lui impersonata e non solo rappresentata e che la sorgente della sua autorità risiedeva nel «diretto mandato divino». Altri punti toccati concernevano la cosiddetta libertà di coscienza, e la «piena libertà di discussione», concetti giudicati ambigui, tali da «ingannare la buona fede di molti uditori poco illuminati». Soprattutto sottolineava il rapporto fra Trattato e Concordato, dal momento che, «secondo la lettera e lo spirito loro, come anche secondo le orali e scritte esplicite intelligenze, sono l'uno completamento necessario dell'altro». Chiara la conseguenza enunciata: «simul stabunt oppure simul cadent anche se dovesse per conseguenza cadere la "Città del Vaticano" col relativo Stato». ${ }^{76}$ Di rincalzo il gesuita Enrico Rosa, sulle pagine della "Civiltà Cattolica", prendeva spunto dalla "parola intrepi-

75 A. CARACCIOLO, Roma Capitale. Dal Risorgimento alla crisi dello Stato liberale, Roma 1956, p. 297. Per questo topos si veda anche ZAMBARBIERI, Roma, "Romanitas, soprattutto pp. 75-79.

76 Il chirografo al card. Gasparri, in “Acta Apostolicae Sedis”, 21 (1929), pp. 297-306. 
da" del pontefice per intessere una comparazione tra quanto accaduto in quei giorni e le vicissitudini riguardanti il concordato napoleonico a più di un secolo di distanza. Si rifaceva al testo dell'allocuzione pronunciata da Pio VII, all'inizio del suo pontificato. Papa Braschi aveva rivolto un appello per ricordare «a tutta la famiglia umana, particolarmente all'Europa civile, la necessità suprema dell'educazione cristiana della gioventù $[\ldots]$, la libertà della Chiesa e il doveroso riconoscimento sociale dei suoi diritti per parte dell'autorità civile». ${ }^{77} \mathrm{Il}$ gesuita si rifaceva all'accordo siglato tra Bonaparte e il Pontefice, reintegrante la religione cattolica in Francia e allo «straordinario plebiscito» del 1802, cui però fecero seguito gli articoli organici, poi estesi all'Italia, assai restrittivi della libertà della Chiesa. Gli effetti delle decisioni del dittatore non durarono perché «di fronte al colosso napoleonico che faceva tremare gli Stati e i monarchi d'Europa» un pio monaco salito al soglio di Pietro «piccolo e debole quanto la forza materiale delle armi [...] fu vincitore del "grande", del forte, del prepotente». Per chi avesse voluto intendere, il parallelo, in verità suggerito anche da altri, ${ }^{78}$ sembrava pronosticare un non radioso futuro per chi si atteggiava a nuovo Napoleone. Il direttore del periodico trattava poi della ratifica dei Patti per la seduta alla Camera, quando «dopo i molti discorsi dei Deputati, secondo le dottrine cattoliche troppo inesatte e talvolta condannabili», si era dovuto ascoltare «per più di tre ore continuate il discorso più inaspettato, in tanta solennità di circostanze, del Capo del Governo». Il quale, sempre secondo il gesuita, non aveva corretto il contenuto della prima allocuzione in quella successiva del 25 maggio, recitata «con più dignità di forma ma con immutata sentenza all'assemblea più scelta del senato». Detto della «gravità immensa dell'ora che passa», concludeva prospettando timori suscitati dall'«ignoranza e la passione contemporanea, congiurate a impedire la divina missione educatrice e pacificatrice della Chiesa, di cui sono parte anche le "rettifiche", non meno che le "ratifiche" degli auspicati Accordi Lateranensi». ${ }^{79}$ Non pochi scalpori dovette

77 Il testo di Pio VII si trova in Bullarium Romanum, XI, Romae 1846, p.1.

78 Nei suoi carnets il card. Baudrillart annotò il 19 maggio: «En sera-t-il de Pie XI et de Mussolini comme de Pie VII et de Napoléon?»: Les carnets du Cardinal Baudrillart, ed. P. Christophe, V, Paris 2003, p. 221.

79 E. ROSA, Tra "Ratifiche" e "Rettifiche". La parola del Papa, in "La Civiltà Cattolica," 1929, III, pp. 97-105. Da rilevare come anche Francesco Luigi Ferrari avesse abbozzato un parallelo tra Mussolini e Napoleone, in un articolo firmato con lo 
suscitare questo articolo, insieme a una misura del prefetto di Roma, che in data 23 luglio ordinerà l'immediato sequestro di tutte le copie del quaderno della rivista «per il contenuto generico e specifico antitaliano e antifascista dell'articolo di fondo intitolato Tra "Ratifiche" $e$ "Rettifiche"». Il biografo di padre Rosa rivelerà che il papa aveva approvato l'articolo, inviando anche all'autore un autografo e una medaglia: ciò, tuttavia, «dovette in quel tempo tenersi prudentemente nell'ombra». ${ }^{80}$ Nuovi attacchi causerà un successivo articolo di p. Rosa, Missione di Ristaurazione sociale dell'educazione e della stampa che, a quanto risulta da informatori vaticani del regime, avrebbe causato l'allontanamento del gesuita in Spagna per qualche mese. ${ }^{81}$ In effetti il direttore della "Civiltà", in quel torno di tempo, aveva ricevuto «truculenti minacce», tra cui quella che lo metteva in guardia dall'insistere nel suo atteggiamento, altrimenti gli sarebbe stata riservata la stessa «fine di don Minzoni». ${ }^{82}$

Qualche distanziamento veniva dunque segnato tra il regime, il pontefice e anche un autorevole opinionista cattolico. Nessuna titubanza invece nutriva, nell'accettare sia i Patti che il movimento mussoliniano, un singolare personaggio del mondo ecclesiastico, Umberto Benigni, fondatore dell'ormai soppresso Sodalitium pianum, associazione, come è noto, risolutamente "integrista", distintasi nel contrastare il modernismo anche ricorrendo a metodi di spionaggio. Qualificato da von Pastor «nemico personale del card. Gasparri», ${ }^{83}$ Benigni dopo la Grande Guerra divenne sia un risoluto sostenitore del fascismo, fiancheggiandolo mediante relazioni strette «et moins simples - come sostiene dopo accurate analisi Émile Poulat- qu'on ne l'observe alors souvent dans le clergé italien», sia «un pourvoyer important de l'antisémitisme italien». ${ }^{84} \mathrm{~A}$ quanto risulta, collaborò con la segreteria particolare del Duce informando sulle questioni ecclesiastiche e in particolare vaticane, anche mediante l'invio di rapporti che pur non stesi con la sua

psedonimo L. SEURRE, L'Eglise et le fascisme. Napoléon et Mussolini, 31 agosto 1931, cit. in L. FERRARI, Scritti dell'esilio, pp. 152-159.

80 A.M. FIOCCHI, Padre Enrico Rosa S.J. Scrittore della Civiltà Cattolica (1870-1938), Roma 1957, p. 191.

81 FIORENTINO, All'ombra di Pietro, pp.178-179 in nota. L'articolo citato nel nostro testo venne pubblicato sulla "Civiltà Cattolica", 1929, III, pp. 3-28.

82 FIOCCHI, Padre Enrico Rosa, p.298.

83 ENGEL-JANOSI, Il Vaticano fra Fascismo e Nazismo, p. 110.

84 É. POULAT, Intégrisme e catholicisme integral, Tournai 1969, p. 62 in nota. 
grafia, «sont bien de sa veine». ${ }^{85}$ Con un senso di grande appagamento salutò la Conciliazione, da lui interpretata non come risultato di quell' "accomodamento", da lui sempre osteggiato, tra Chiesa e società moderna, ma come punto d'arrivo di un processo che egli stesso aveva contribuito ad alimentare. Poco tempo dopo la firma dei Patti, pubblicò, sul bollettino a diffusione internazionale "Veritas", il proprio giudizio riguardante gli accordi: per lui rappresentavano «la démonstration que le fascisme avait su reussir où le démo-libéralisme avait souvent échoué et grâce à lui, par-dessus tout, infiniment plus grandiose, Rome enfin réconcilié avec elle-même». La lotta delle investiture era ormai liquidata e non sarebbe stata più ripresa. Incontrando Mussolini, la Chiesa si era felicemente imbattuta in un nuovo Costantino. Dopo la Roma imperiale che aveva civilizzato il mondo, e dopo la Roma dei pontefici che l'aveva reso cristiano, ecco che una nuova Roma, nata dopo la marcia sulla capitale, inaugurava il modello di una nuova civiltà. Percorso ideale, dunque, e strada obbligata per il futuro: «la route par laquelle l'Italie agonisante s'est sauvée en 1922, tous y passeront». ${ }^{86}$

Di stile e contenuti molto diversi, e inaspettati, tenuto conto delle vicissitudini del personaggio, furono le annotazioni sui Patti di Ernesto Buonaiuti. Conviene sostarvi un poco, sia per il loro carattere singolare sia per gli spunti suggestivi che vi si trovano. Prete "romano", come amava definirsi, notevolissimo studioso di storia del cristianesimo, professore all'Università La Sapienza di Roma dall'ottobre 1916, conobbe aspre traversie nei rapporti con le autorità ecclesiastiche. Sospettato di modernismo durante il pontificato di Pio X, sospeso a divinis e scomunicato con decreto del 14 gennaio 1921, dopo la parentesi di una reintegrazione ecclesiastica, incorse tre anni dopo nuovamente nella stessa

85 É. POULAT, Catholicisme, démocratie et socialisme. Le mouvement catholique et Mgr. Benigni de la naissance du socialisme à la victoire du fascisme, Tournai 1977, p. 459 in nota. Per i rapporti consegnati alla Segreteria del Duce si veda SCOPPOLA, Chiesa e fascismo, Bari 1971, pp. 145-149.

86 "Veritas", 6 (1929), p.1 (il numero della rivista uscì in data 26 ottobre). Si veda in proposito POULAT, Catholicisme, démocratie et socialisme, pp.464-465. Su Benigni fondamentali sono le due opere appena citate di Poulat, dove la precisa ricostruzione biografica del personaggio rientra in un'ampia ed accurata disamina dell'evoluzione del cattolicesimo tra Otto e Novecento. Per altre reazioni ai Patti nel mondo intransigente si veda M. TAGLIAFERRI, L' "Unità Cattolica". Studio di una mentalità, Roma 1993, pp. 294-297. 
condanna, e infine con decreto del 25 gennaio 1926 fu dichiarato «excommunicatum et expresse vitandum». Il suo magistero presso l'ateneo romano venne seguito con circospetta diffidenza e non celata ostilità da esponenti della curia vaticana. ${ }^{87}$ Insistite si moltiplicarono le critiche a lui rivolte da cattolici e specialmente dal p. Rosa, e ripetuti furono anche i tentativi della S. Sede per destituirlo dall'insegnamento, attraverso approcci con esponenti governativi. Anzi, durante le trattative in vista della Conciliazione, il suo ruolo di docente diventò oggetto del contendere tra i negoziatori vaticani e governativi, in un'altalenante dare-avere per ciò che dalle due parti veniva postulato: quella cattedra era riguardata, scriverà più tardi don Ernesto, «quale arma di combattimento [...], come posta di ricatto in una controversia politica». ${ }^{88} \mathrm{Le}$ cadenze di quelle discussioni sono state ricostruite in modo dettagliato, fino all'approdo concordatario. ${ }^{89}$ Né Buonaiuti si nascose, in base a notizie pervenutegli, che «qualcuna delle clausole stilizzate nei commi del Concordato» erano state scelte appositamente per colpirlo. Infatti un'informazione da lui avuta riguardava quella disposizione concordataria in forza della quale agli ex preti, irretiti da censure, era impedito di occupare posti a contatto con il pubblico..$^{90}$ In effetti ciò appare in un comma dell'art. 5 del Concordato, quantunque per il momento la questione rimanesse impregiudicata. ${ }^{91}$ In realtà un altro articolo, il 29, comma $i$, affidava al Governo italiano l'esecuzione delle sentenze ecclesiastiche, dunque anche la disposizione che privava dell'abito sacerdo-

87 Tra le molte indicazioni bibliografiche basti qui rinviare alle notizie in proposito che offre la sintesi, accurata pur nella brevità, di F. PARENTE, Ernesto Buonaiuti, Roma 1971, spec. pp. 59-70.

88 Pellegrino di Roma, p.263.

89 MARGIOTTA BROGLIO, Italia e S. Sede, pp.171-180; dello stesso autore, Ernesto Buonaiuti, in "Storia contemporanea" 2 (1971), pp. 803-823. L. BEDESCHI, Buonaiuti, il Concordato e la Chiesa, Milano 1970, dove le vicende sono esaminate con dovizia di particolari e con l'appoggio della corrispondenza tra Buonaiuti, Guido Cagnola e Luigi Bietti. Si veda anche, oltre alla citata sintesi di Parente, F. MARGIOTTA BROGLIO, Ernesto Buonaiuti, in Modernismo, Fascismo, Comunismo, pp. 142-148, e pure l'ottimo saggio di una intelligente studiosa, prematuramente scomparsa, L. GIORGI, Il "Caso Buonaiuti" e il Concordato, in "Il Ponte", 25, nn. 2-3 (28 febbario21 marzo 1979: estratto di 48 pp.).

90 Oltre al testo del Concordato, si veda quanto Buonaiuti scrisse a Cagnola in data 23 febbraio 1929, in BEDESCHI, Buonaiuti, il Concordato e la Chiesa, p.397.

91 MARGIOTTA BROGLIO, Italia e S. Sede, p. 179. 
tale gli ecclesiastici colpiti da censura. ${ }^{92}$ Tuttavia in quel febbraio del 1929, dopo le spiegazioni fornite dal duce alla Camera e al Senato, Buonaiuti intravide la possibilità che si sciogliessero gli impedimenti di cui le autorità ecclesiastiche avrebbero potuto avvalersi per limitare o addirittura cancellare la sua libertà accademica. Fu questa la sensazione, insieme all'assicurazione data da Mussolini circa la non retroattività dell'art. 5 del Concordato, che forse lo indusse a elogiare gli interventi del capo del governo, tanto più che i riferimenti del discorso mussoliniano agli studi sulle origini cristiane e in particolare sulla figura di S. Paolo sembra siano stati forniti al Duce dallo stesso sacerdote romano, ${ }^{93}$ forse tramite Mario Missiroli. ${ }^{94}$ Anche l'asserto sulla pretesa stagnazione degli studi religiosi in Italia, poté sembrargli utile a incentivare le ricerche storico-critiche sul cristianesimo primitivo e sul cristianesimo toutcourt, oltre naturalmente a prospettare una slargata libertà di ricerca in tale settore. Questo convincimento espresse sia in un articolo giornalistico che gli creò difficoltà con gli amici ${ }^{95}$ sia in una versione più impegnativa, data la sede su cui la pubblicava, vale a dire la rivista scientifica da lui diretta, nel numero di luglio 1929. Con una nota firmata La Direzione, ma sicuramente sua, mirava a dissipare l'equivoca estensione interpretativa della Conciliazione. Essa non determinava a suo dire l'«asservimento della spiritualità italiana oltre che ai valori centrali del cattolicismo anche ai voleri della burocrazia inquisitoriale». Di qui l'elogio alla «geniale arditezza» del Capo del Governo che aveva ricavato «dai dati dell'indagine storica intorno al processo di sviluppo del messaggio evangelico una lucida e istruttiva lezione di storia», garantendo così «in maniera categorica l'inviolabile libertà dell'indagine scientifica $\mathrm{e}[\ldots]$ anche il rifiorire della cultura cattolica in Italia». ${ }^{96}$ Sul piano per-

92 GIORGI, Il "Caso Buonaiuti”, pp. 23-24.

93 L'indiscrezione deriva da una confidenza che il giornalista Emanuele Barabino fece a mons. Alfred Baudrillart il 4 ottobre 1929 ( Les carnets du Cardinal Baudrillart, p. 331).

94 BEDESCHI, Buonaiuti, il Concordato e la Chiesa, p. 158.

95 L'articolo, con il titolo Gli studi religiosi in Italia ,venne pubblicato su " Il Corriere Padano”, 31 maggio 1929, cit. in PERTICI, Chiesa e Stato, p. 189 n.60. Per i dissapori con Cagnola e con don Bietti, si veda BEDESCHI, Buonaiuti, il Concordato e la Chiesa, pp. 154-159.

96 La Direzione, Cattolicismo e cultura in Italia, in "Ricerche religiose", 5 (1929), pp. 23-24. 
sonale nutrì più di una speranza verso la sua piena reintegrazione all'attività didattica, al punto da indirizzare a Mussolini, in data 2 luglio, una lettera nella quale, rievocate le peripezie del suo insegnamento universitario e appellandosi alle «solenni e memorande parole» pronunciate dallo stesso duce alla Camera dei Deputati e al Senato, gli chiedeva di poter riprendere le lezioni sulla cattedra di storia del cristianesimo, l'unica nelle università italiane, e quindi sentendosi il solo nel paese «a rappresentare e a tutelare ufficialmente» la disciplina «la più delicata e la più viva del fascio di quegli studi storico-religiosi che fuori d'Italia costituiscono la parte più cospicua della cultura morale». ${ }^{97} \mathrm{Il}$ comportamento di Buonaiuti e in particolare la missiva a Mussolini, sono verosimilmente interpretabili con la sua dedizione alla ricerca e al rapporto comunicativo con i giovani universitari, poiché, come è stato giustamente notato, egli provava una «necessità quasi fisica di insegnare». ${ }^{98}$ D'altro canto, secondo Ambrogio Donini, che era stato tra i suoi più fedeli discepoli, quelle avances verso il capo del fascismo costituivano «una delle manifestazioni più emblematiche della sconfinata ingenuità» del maestro, così da palesarne anche l'«incomprensione totale della politica fascista nei confronti della Chiesa».9 Altri erano i suoi intendimenti: per esempio, due anni prima degli accordi del Laterano egli, scrivendo a Giuseppe Rensi, aveva rimarcato il «divario incolmabile» tra vita religiosa e vita politica che gli era sembrato di intravvedere in un atteggiamento mussoliniano non pregiudizievole per l'autonomia di coscienza dei credenti. ${ }^{100}$

Il miraggio della salvaguardia della propria professione didattica e scientifica, svanirà lentamente. Ma egli non smise di ribadire, proprio verso la fine del 1929 una costante tipica nella traiettoria del suo pensiero. Scrivendo a proposito dell'opera agostiniana De Civitate Dei, vi

97 La lettera è pubblicata da GIORGI, Il "Caso Buonaiuti”, pp. 40-41.

98 MARGIOTTA BROGLIO, Ernesto Buonaiuti, p.139, si veda anche p. 143.

99 Così Donini in una nota alla pubblicazione da lui curata del carteggio di Buonaiuti con Remo Missir: E. BUONAIUTI, La vita allo sbaraglio. Lettere a Missir, Firenze 1980, p.123. Si tratta di una postilla alla lettera di Buonaiuti a Missir, in data 26 maggio 1929. Ma è da notare come don Ernesto, cinque giorni dopo la proclamazione dei Patti, non avesse nascosto al medesimo corrispondente i propri timori per la propria «situazione accademica ed extra-accademica» (lettera del 17 febbraio 1929, ibid. pp.110-111).

100 Lettera di Buonaiuti a Giuseppe Rensi del 2 giugno 1927, pubblicata da A. Vigorelli, Lettere a Giuseppe Rensi (1898-1939), in Fondo Giuseppe Rensi. Inventario con una scelta di lettere inedite, a cura di L. Ronchetti e A. Vigorelli, Milano 1996 p.275. 
leggeva non solo e non tanto un'identificazione della Città di Dio con la comunità ecclesiatica, ma soprattutto un «concetto primordiale ed essenziale» in grado di situare la Civitas Dei «prima e al di là della Chiesa [...], nata potenzialmente in contrasto con la Civitas terrena». Ne scaturiva «un esempio e un ammonimento», che lo studioso, superando i confini della critica storica, traduceva nell'apostrofe finale: «Guai all'uomo e alla Chiesa il giorno in cui, per la coerenza astratta e il successo empirico, pongano a repentaglio l'irresolubile paradossalità della superiore giustizia». ${ }^{101}$ Anche l'intesa concordataria, ritenuta un successo, veniva dunque relativizzata, e la presenza dei credenti nella società era vista come quella «paradossale cittadinanza» che Buonaiuti aveva evocato anni prima, pubblicando la traduzione dell' $A$ Diogneto, testo cristiano risalente all'inoltrato II secolo della nostra era: egli vi scorgeva l'ideale di una comunità dei credenti che avessero adottato lo stile descritto nelle antiche parole: «Vivono nella loro patria ma come forestieri; partecipano a tutto come cittadini, e da tutto sono distaccati come stranieri». ${ }^{102} \mathrm{Su}$ questo sfondo, gli accordi con il potere politico rischiavano di confezionare quella "camicia di Nesso", che era il nazionalismo, cioè «il centauro della modernità», capace di ridurre il cristianesimo a «una federazione di chiese nazionali», cancellandone la fon-

101 E. BUONAIUTI, Intorno al De Civitate Dei, in "Ricerche religiose", 5 (1929), pp. 481-493, cit. alle pp. 492-493. Il fascicolo riporta la data del novembre dello stesso anno. Già nell'agile profilo da lui dedicato al vescovo di Ippona, Buonaiuti aveva formulato sinteticamente la medesima interpretazione, condensandola anche nella frase: «Fra la Chiesa e la società di Dio non v'è alcuna identità di confini cronologici o spaziali» (E. BUONAIUTI, S. Agostino, Roma 1917, cito dall'edizione più recente curata da A.MODA, Cosenza 1996, p. 88 (il testo è quello della seconda edizione del 1923), tradotta anche in romeno (M. RAVÀ, Bibliografia degli scritti di Ernesto Buonaiuti, Roma 2015, p. 29: si tratta di una ristampa anastatica della prima edizione, Firenze 1951). Per questo importante tema agostiniano tra i molti studi segnalo i sempre validi É. GILSON, Eglise et cité de Dieu chez Saint Augustin, in "Archives d'histoire doctrinale et littéraire du Moyen-Age", 20 (1953), pp.5-23., Y. M. CONGAR, Civitas Dei et "Ecclesia” chez Saint Augustin, in "Revue des Études Augustiniennes”, 3 (1957), pp. 1-14; si veda la rassegna su contributi più recenti di PH. CURBELIÉ, Les études sur La cité de Dieu, in "Revue d'ètudes augustiniennes et patristiques", 50 (2004), pp. 311-329. Dello steso autore è da vedere il volume, La Justice dans "La Cité de Dieu”, Paris 2004.

102 Lettera a Diogneto. Testo, traduzione e note a cura di Ernesto Buonaiuti, Roma 1921. Da notare come l'edizione buonaiutiana venisse elogiata da Hans von Soden, in "Theologische Literaturzeitung", 28 (1924), coll. 516-518. 
damentale natura di «carismatica aggregazione supernazionale nell'anagrafe del Regno di Dio». ${ }^{103}$ Quando Buonaiuti vergava queste parole, da circa un anno gli era stato imposto da parte dell'autorità civile, in esecuzione della sentenza ecclesiastica, l'abbandono della veste talare, ai sensi del citato art.29 del Concordato, che comminava «le stesse sanzioni con le quali si puniva l'uso abusivo della divisa militare». Fu per lui un duro colpo, commentato sulla sua rivista in maniera sarcastica verso il tribunale della Curia evocando antichi testi cristiani, nei quali non solo il sacerdozio, ma anche la professione evangelica, erano considerati «come una milizia irrevocabile e sacrosanta, importante doveri e diritti, superiori ad ogni mansione, ad ogni connotazione, ad ogni controllo burocratico». ${ }^{104}$ Pur deponendo l'abito e sforzandosi di seppellire dispiaceri e disillusioni, avrebbe voluto continuare la sua missione di docente e di studioso, ché era per lui «milizia e apostolato»,

103 e.b., La camicia di Nesso, in "Ricerche religiose", 7 (1931), p.393. L'osservazione riguardante il frazionamento nazionalistico era già presente in una nota buonaiutiana scritta prima degli accordi lateranensi: «Il cattolicesimo non è più una famiglia carismatica, solidale nello Spirito che è il Signore e nella ricerca insonne del suo unico Regno nella pace, nella carità e nella giustizia; è piuttosto una federazione di chiese nazionali strette nei rapporti di una organizzazione burocratica» : L'universalità cristiana, in "Ricerche religiose", 4 (1928), p. 264. Da rimarcare qui anche l'interpretazione buonaiutiana del detto, allora echeggiato nel titolo del libro ricco di documenti, pubblicato da M. MISSIROLI, Date a Cesare, Roma, Libreria del Littorio, 1929: il logion "date a Cesare"non prospettava una visuale in grado di fissare il confine delle rispettive competenze di Chiesa e Stato. In effetti, come è stato opportunamente rilevato, indicava «un'opposizione tra due orizzonti radicalmente discontinui, quello di Dio e quello del potere umano, non un criterio di delimitazione di un confine giuridico tra due sfere di legittimità; esso non può venire così assunto a giustificazione né di una qualche forma di religione civile, né di civiltà religiosa [...]. La risposta a quale sia il contributo da versare a Cesare e a Dio attraversa necessariamente la coscienza di ciascun uomo e la sua libertà» (M. RIZZI, Cesare e Dio. Potere spirituale e potere secolare in Occidente, Bologna 2009, p.219). Per i ritorni di Buonaiuti sul tema, si vedano ad es. Dio e Cesare, in "Ricerche religiose", 9 (1933), pp. 568-569; Cesare e Cristo , in "Religio" (1939), p.311. Proprio pochi mesi dopo la Conciliazione era uscito il già citato libro di MISSIROLI, Date a Cesare. Per altri riferimenti di Buonaiuti alla prassi concordataria si veda il suo volume Pio XII, Firenze 1958 ( $2^{\mathrm{a}}$ ed, la prima era uscita nel 1946), pp. 47-61; 79-129. La breve riflessione sul logion «Date a Cesare» alle pp. 300302; Pellegrino di Roma, pp. 265-272.

104 e.b. "Militia Christi", in "Ricerche religiose," 6 (1930), pp. 179-180. Sulla vicenda Buonaiuti lasciò un duro commento nell'autobiografia Pellegrino di Roma, pp. 271-276. 
come scriveva pochi mesi dopo in un'appassionata recensione del volume di Albert Schweitzer, Die mystik des Apostels Paulus. ${ }^{105}$

Di lì a poco non gli fu possibile continuare l'insegnamento universitario. Infatti venne estromesso, con decreto del 28 dicembre 1931, dal corpo accademico, avendo rifiutato di prestare il giuramento, in forza del quale i docenti dovevano impegnarsi a formare «cittadini operosi, probi e devoti alla Patria e al Regime Fascista». Fu dunque tra i pochi professori universitari sottrattisi a quell'atto. Il suo gesto rispecchiava, come scriverà nell'autobiografia, il proposito di rimanere fedele alle basilari prescrizioni della professione cristiana e in sostanza ad una linea di condotta che gli impediva di «mescolare preoccupazioni di indole politica contingente alla questione morale che il giuramento comportava»: una personale conferma, dell'interpretazione che aveva fornito a proposito dell'ideale da lui letto nell'agostiniana Civitas Dei, e una concezione del rapporto tra politica e coscienza che considerava la religiosità come «fermento invisibile della vita associata». ${ }^{106}$

È agevole notare il divario tra le valutazioni di Buonaiuti e quelle di Umberto Benigni, che, com'è noto, lo aveva istruito parecchi anni prima, come insegnante al seminario dell'Apollinare, sugli strumenti dell'indagine critica. ${ }^{107} \mathrm{Ma}$ pure, si constata la disparità di giudizi a confronto con quelli di Rosa, il suo confutatore più determinato e per certi versi spietato. ${ }^{108}$ Enrico Rosa, Umberto Benigni, Ernesto Buonaiuti: figure rappresentative di tre correnti che sembrarono ad Antonio Gramsci ben connotare le «tre tendenze organiche del cattolicismo» di quel tempo, rispettivamente, «gesuiti, cattolici integrali, modernisti». L'acuto pensatore, constatando la presenza di tali orientamenti tra cattolici, era portato a ritenere che la forza coesiva della Chiesa fosse minore di quella che

105 Il volume di Schweitzer era uscito a Tübingen nel 1930; la recensione buonaiutana, in "Ricerche religiose", 6 (1930), pp.301-303.

106 Pellegrino di Roma, p.278: notizie essenziali in PARENTE, Ernesto Buonaiuti, pp. 79-81 e MARGIOTTA BROGLIO, Ernesto Buonaiuti, in Modernismo, fascismo, comunismo, pp.147-153. (anche per i controlli successivi del regime nei confronti di Buonaiuti).

107 A. ZAMBARBIERI, Il cattolicesimo tra crisi e rinnovamento. Emesto Buonaiuti e Enrico Rosa nella prima fase della crisi modernista, Brescia 1979, pp.149-154.

108 Si veda il volume citato alla nota precedente e L. BEDESCHI, Il processo del Sant'Uffizio contro i modernisti romani, in " Centro Studi per la storia del Modernismo. Fonti e documenti”, 7 (1978), pp. 7-118. 
si potesse presumere, nonostante gli sforzi della gerarchia ecclesiastica sia per rafforzarla che per mostrarla sulla scena mondiale. ${ }^{109}$

In effetti la Conciliazione aveva fornito al papato la garanzia statuale della Città del Vaticano, una Delo cioè, per riprendere l'immagine di von Döllinger, emersa da fluttuanti trattative politico giuridiche. Insieme sanciva un'intesa di vertice tra Chiesa e fascismo, diramantesi nelle stratificazioni sociali, che determinava convergenze anche nelle modalità di comunicazione, fino a creare una caratteristica koiné ideologica. ${ }^{110}$ Tuttavia il panorama risultava variegato, refrattario a una troppo automatica identificazione tra cattolicesimo e regime. Dinamismi autonomi, suscettibili di esplicarsi sul lungo periodo, erano attivati sia dall'associazionismo cattolico ${ }^{111}$ sia da movimenti culturali promossi in ristrette cerchie elitarie, ${ }^{112}$ nonché dalle pratiche consuetudinarie di culto, con l'universo simbolico che le supportava. ${ }^{1{ }^{13}}$ Un connubio tra il regime e la compagine dei cattolici, parve abbastanza scontato anche negli addentellati con alcune devozioni, che sovrapponevano festività fasciste e liturgie cristiane. Ma il tentativo della dittatura di configurare

109 A.GRAMSCI, Cattolici integrali, gesuiti, modernisti, Quaderno 20 (XXIV), par.4. Gramsci sostenne che dopo la Conciliazione si verificò una ripartizione del lavoro culturale fra casta cattolica e intelligenza laica: la scuola era la sede principale del compromesso tra Chiesa e regime; alla prima era riservata l'area delle scuole elementari e medie, dove «si plasmano le conoscenze», alla seconda quella dell'insegnamento superiore e universitario per la formazione della classe dirigente (C. VASALE, Politica e religione in A. Gramsci. L'ateodicea della secolarizzazione, Roma 1979, p.139.) Si vedano pure F. FROSINI, L'egemonia e il "subalterno": utopia, religione, democrazia, in G. COSPITO - F. FROSINI, Egemonico/subalterno, numero monografico della rivista " International Gramsci Journal” , 2/4 (2016), pp. 126-166; son da vedere tutti i saggi contenuti nel numero e in particolare quello di G. FRANCIONI, Un labirinto di carte (introduzione alla filologia gramsciana), pp-7-48. Sul problema religioso in Gramsci sempre utile H. PORTELLI, Gramsci et la question religieuse, Paris 1971. Sulle preoccupazioni unitarie della gerarchia cattolica si veda quanto scrive A. GALASSO, L'altra Europa. Un'antropologia storica del mezzogiorno d'Italia, Napoli 2009, pp. 441-443.

110 R. MORO,Nazione, cattolicesimo e regime fascista, in "Rivista di storia del cristianesimo", 1 (2004), pp.129-147.

111 F. TRANIELLO, L'Italia cattolica nell'era fascista, in G. DE ROSA, Storia religiosa dell'Italia. III L'età contemporanea, Roma-Bari 1993, pp.275-278.

112 MORO, La formazione della classe dirigente cattolica (1929-1937), passim.

113 Ottime osservazioni di G.BATTELLI, Santa Sede e vescovi nello Stato unitario. Dal secondo Ottocento ai primi anni della Repubblica, in G. CHITTOLINI - G. MICCOLI (edd.), Storia d'Italia. Annali 9. Chiesa e potere politico, Torino 1986, p.846. 
una propria religione dovette arrestarsi «là dove la sacralizzazione fascista della politica s'imbatté in modo inconciliabile con la forma cattolica di sacralizzazione della religione». ${ }^{114} \mathrm{Di}$ fatto la forza di tradizioni secolari, insieme a linguaggi e riti entrati stabilmente nelle consuetudini, e quella che si suol chiamare "religiosità popolare", non a caso problematicamente considerata anche da Antonio Gramsci, ${ }^{115}$ creavano un universo mentale forse in parte scalfibile ma non sopprimibile. Apparteneva, per usare la terminologia di uno studioso quale Alphonse Dupront, a quelle «puissances et latences», ${ }^{116}$ che, nei riverberi sociopolitici, contribuiscono alla modellazione della società. Ma ciò sconfina in un perimetro molto più ampio di quello che il Trattato e il Concordato allora e si dischiude su diverse e molteplici dimensioni spaziali e temporali. Cioè, per dirla in modo abusato, su una più durevole e coinvolgente Storia.

\section{RINGRAZIAMENTI}

Intendo porgere un sentito ringraziamento agli amici prof. Marco Barbieri e Italo Colombo per il loro molto intelligente e paziente aiuto nella trascrizione e nella revisione del testo.

114 L. KLINKHAMMER, Il fascismo italiano tra religione di Stato e liturgia politica, in U. FERRONE (ed), La Chiesa cattolica e il totalitarismo, Firenze 2004, pp. 185-203, cit. p. 202. Si veda supra n. 26, Sarebbero da studiare le componenti comuni e quelle differenziali, per quanto riguarda le direttive e i comportamenti nelle rispettive forme di sociabilità proposte dal regime e dai cattolici. Qualche osservazione si può trovare nel mio saggio La gioventù di Azione Cattolica nel Lodigiano durante alcuni anni del fascismo, in P. PECORARI (ed), Chiesa, Azione Cattolica e fascismo nell'Italia settentrionale durante il pontificato di Pio XI (1922-1939), Milano 1979 pp. 603-626, spec. 625-626.

115 Si veda ad esempio T. LA ROCCA, Gramsci e la religione, Brescia 1991, pp.28-29 (l'autore sottolinea l'importanza attribuita da Gramsci al rapporto tra senso comune e religione, diversa nei ceti popolari rispetto sia da quella degli intellettuali sia da quella organicamente sistemata dalla gerarchia ecclesiastica). Da vedere anche J. WAINWRIGHT, On Gramsci's conceptions of the worlds, in "Transactions of the British Geographers", n.s., 2010, pp. 507-521.

116 E il titolo di un suggestivo volume di A. DUPRONT, Puissances et latences de la religion catholique, Paris 1993, in cui l'autore, attingendo alla filosofia, alla psicologia, all'antropologia e naturalmente alla storia, formula ipotesi circa l'impatto del cattolicesimo sulla società attuale. Su questo autore si veda S.H. FRANCESCHI, Les intermittences du temps. Lire Alphonse Dupront, Paris 2014. 